\title{
SINGLE-BEAM COHERENT INSTABILITIES IN CIRCULAR ACCELERATORS AND STORAGE RINGS
}

C. Pellegrini

April 1982

\section{ACCELERATOR DEPARTMENT}

\author{
BROOKHAVEN NATIONAL LABORATORY \\ ASSOCIATED UNIVERSITIES, INC. \\ UPTON, NEW YORK 11973 \\ UNDER CONTRACT NO. DE-AC02-76CH00016 WITH THE \\ UNITED STATES DEPARTMENT OF ENERGY
}




\section{DISCLAIMER}

This report was prepared as an account of work sponsored by an agency of the United States Government. Neither the United States Government nor any agency Thereof, nor any of their employees, makes any warranty, express or implied, or assumes any legal liability or responsibility for the accuracy, completeness, or usefulness of any information, apparatus, product, or process disclosed, or represents that its use would not infringe privately owned rights. Reference herein to any specific commercial product, process, or service by trade name, trademark, manufacturer, or otherwise does not necessarily constitute or imply its endorsement, recommendation, or favoring by the United States Government or any agency thereof. The views and opinions of authors expressed herein do not necessarily state or reflect those of the United States Government or any agency thereof. 


\section{DISCLAIMER}

Portions of this document may be illegible in electronic image products. Images are produced from the best available original document. 


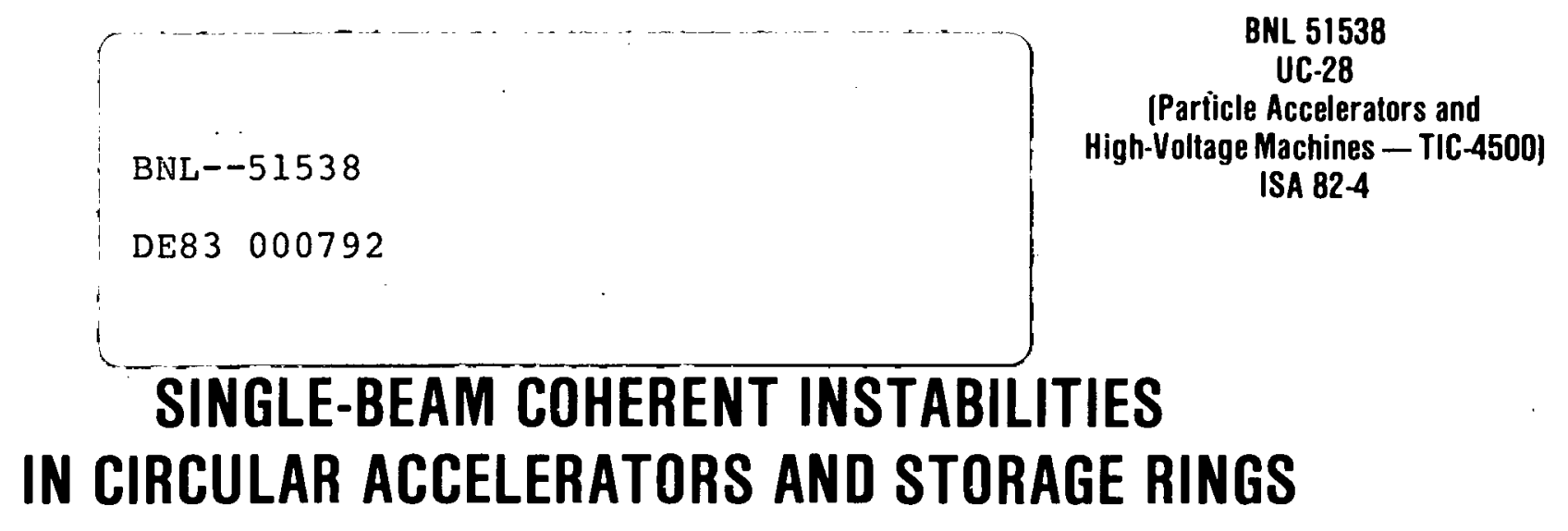

C. Pellegrini

April 1982

ACCELERATOR DEPARTMENT

BROOKHAVEN NATIONAL LABORATORY

UPTON, NEW YORK 11973 


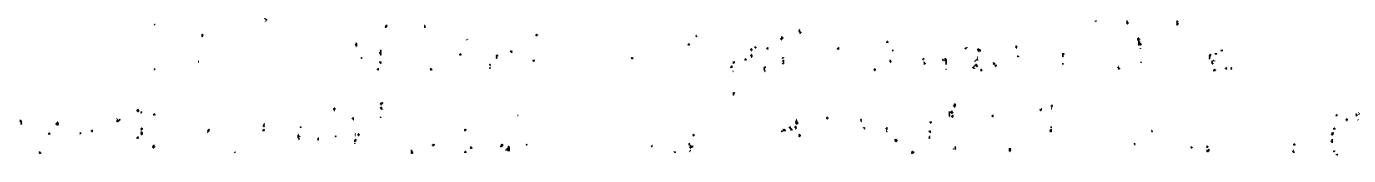

\section{DISCLAIMER}

This report was prepared as an account of work sponsored by an agency of the United States (invernment Neither the United States Governmenil ।.1! ally ayency thereot, nor any of their employees, nor any of their contractors, subcontractors, or their employees, makes any warranty, cxpress or imulied. or assımes any leg? lianility or responsibility for the accuracy, completentess, or usefulness of any information, apparatus, product, or process disclosed, or represents that its use would not infringe privately owned rights. Reference herein to any specific commercial product, process, or service by trade name, trademark, manufacturer, or

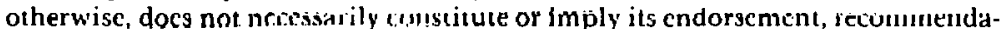
tion, or favoring by the United States Government or any agency, contractor or subcontractor thereof. The views and opinions of authors expressed herein do not necessarily state or reflect those of the United States Government or any agency, rontractor or subcontractur thercof.

Printed in the United States of America Available from

National Technical Information Service

U.S. Department of Commerce 5285 Port Royal Road Springfield, VA 22161

NTIS price 'codes:

Printed Copy: A04; Microfichc Copy: A01 
THIS PAGE

\section{WAS INTENTIONALLY \\ LEFT BLANK}




\section{Abstract}

The theory of the stability of high intensity particle beams in circular accelerators and storage rings is developed. A simple one dimensional oscillator system is used to introduce the discussion of the stability problem and of the effect of a frequency distribution. These results are then extended to the case of a system of particles executing synchrotron and betatron oscillations in a circular accelerator. In the absence of a general solution of the stability problems, it is convenient to introduce a classification of the. instabilities, using the characteristic times and lengths of the particle system. This allows us to identify a set of parameters of the particle beam for which one can establish the stability conditions. 


\section{CONTENTS}

Sections $\quad \underline{\text { Page }}$

1. Introduction ......................................

2. The Stability Problem.................................

3. The Vlasov Equation.................................

4. Equations of Motion for the Longitudinal Degree of Freedom........ 12

5. Equations of Motion for the Transverse Oscillations.............15

6. The Transverse and Longitudinal Collective Forces............... 18

7. The Mode1 Impedance..................................

8. Longitudinal Instabilities of a Coasting Beam................. 33

9. Longitudinal Instabilities of Bunched Beams.................. 40

10. Transverse Instabilities of a Coasting Beam................ 55

11. Transverse Instabilities of Bunched Beams.................. 58

References.......................................66 


\section{Introduction}

Coherent instabilities of particle beams in circular accelerators and storage rings have been the subject of many theoretical and experimental works, starting from the early 1960s. I will not try to review all of these, but will cite only the most recent or those more strictly related to the particular way I describe these phenomena. An excellent review of the early work is given in reference 1 and of some aspects more related to storage rings, in reference 2 .

The coherent instabilities to be discussed are produced by the electromagnetic interaction of the charged particle beam with the walls of the vacuum chamber in which the beam is moving. The wall geometry is complicated by the presence in the accelerator of the diagnostic equipment, radio-frequency cavities, and other equipment needed to operate the system. The field produced by the beam and modified by the walls causes a force, proportional to the beam current, that acts on the beam and can lead to what we call a coherent instability.

Since the wall geometry can be very different for the various accelerators, it is useful to follow the idea of Sessler and Vaccaro(3) of characterizing the vacuum chamber and all the associated devices, called the "beam environment," by an impedance function $z(\omega)$, relating the beam current and the electromagnetic field perturbing the beam. In this way one can study the beam dynamics in general terms and then apply the results to a particular accelerator by specifying the impedance.

Although one can write the general equations describing the beam dynamics and the stability properties, it is not possible to obtain a general solution of these equations for a realistic beam charge distribution and wall impedance. It is, however, possible to obtain analytical solutions valid in certain regions of the parameter space of this problem and numerical solutions in the more complicated cases.

It is possible to think of instability mechanisms different from the beam wall interaction considered here. One example is the two-species instability, which can occur, for instance, in a proton beam partially neutralized by electrons produced by ionization of the residual gas in the vacuum chamber. The electrons and protons can execute coherent oscillations of growing amplitude leading to beam loss. Although these effects can be important under some conditions, they will not be discussed here.

The paper is organized as follows. In Sections 2 and 3 we discuss, using a simple example, what we mean by coherent instabilities and illustrate the techniques that can be used to study this problem. In particular, we introduce the Vlasov equation and show how it can be used to evaluate the stability properties. In Sections 4 and 5 we consider a particle beam and introduce the equations of motion for the case of longitudinal and transverse coherent oscillations. In Sections 6 and 7 we derive an expression for the longitudinal and transverse coherent forces. These forces are then used in the vlasov equations to obtain a dispersion relation and define the limiting conditions for stability (threshold current). These threshold conditions are obtained for several different sets of beam parameters, corresponding to what we call slow or fast 
instabilities and long or short wavelength of the perturbation. The longitudinal stability is discussed in Sections 8 and 9 and the transverse stability in Sections 10 and 11 .

\section{The Stability Problem}

We consider a set of particles, for instance a beam of an accelerator, and assume that for this system there exists an equilibrium state. We want to know whether a small perturbation around the equilibrium state will grow (instability) or decay (stability).

As an example let us consider a set of one-dimensional oscillators subject to a collective force proportional to the center-of-mass displacement. We can write the equation of motion for particle $l$ as

$$
\ddot{x}_{\ell}+\omega_{\ell}^{2} x_{\ell}=N \int G\left(t-t^{\prime}\right) D\left(t^{\prime}\right) d t^{\prime}
$$

where

$$
D(t)=\frac{1}{N} \sum_{\ell=1}^{N} x_{\ell}
$$

and we assume the collective force to be proportional to the number of particles, $\mathrm{N}$.

The Green function $G(t)$ must satisfy the causality condition, $G(t)=0$ if $t<0$. This function describes the memory of the system producing the collective force. In the simplest case, in which the system has no memory, all interactions are instantaneous in time and $G\left(t-t^{\prime}\right)=\delta\left(t-t^{\prime}\right)$.

An example of a system with memory is a resistive metallic wall. The fields produced by the charges and currents induced by an electron passing near the wall can last long after the particle has moved away.

We have considered the possibility that the particles have different oscillation frequencies, $w_{\ell}$. A difference in frequency $c$ an be produced either by a variation in the energy, $\Delta E$, of the particles or by the action of nonlinear forces, which we have neglected in writing Eq. (1). In the second case the frequency depends on the particle oscillation amplitude,

$$
\omega_{\ell}^{2}=\omega^{2}\left(\Delta \mathrm{E}_{\ell},\left|\mathrm{x}_{\ell}\right|\right)
$$

We assume that the variation in frequency is small

$$
\frac{\Delta \omega_{\ell}}{\omega_{\ell}} \ll 1
$$

and call $\omega_{0}$ the central value of the frequency distribution. 
For our set of oscillators the equilibrium state is that in which the center of mass does not move and $D(t)=0$. We now perturb the system by displacing the center of mass. To study the motion that follows the perturbation, we look for a solution of Eq. (1) of the form

$$
\begin{gathered}
x_{\ell}=A_{\ell} e^{-i \Omega t}, \\
D=\bar{A} e^{-i \Omega t}, \\
\bar{A}=\frac{1}{N} \sum_{\ell=1}^{N} A_{\ell},
\end{gathered}
$$

such that all particles oscillate coherently with the same frequency. Substituting in Eq. (1) and introducing the "impedance" function

$$
i z(\omega)=\int d t G(t) e^{+i \omega t}
$$

we obtain

$$
\left(\omega_{\ell}^{2}-\Omega^{2}\right) A_{\ell}=2 \pi i \bar{A} N Z(\Omega)
$$

A solution of Eq. (9) can be obtained easily if it is assumed that $\omega_{\ell}$ is not a function of $A_{l}$. The case in which $w_{l}$ is a function of $A_{l}$ is more complicated and will be studied in the next section with the help of the Vlasov equation. From Eq. (9), dividing by $\left(\omega_{\ell}^{2}-\Omega^{2}\right)$ and summing over all particles, we obtain a dispersion relation

$$
1=2 \pi i N Z(\Omega) \frac{1}{N} \sum_{\ell=1}^{N} \frac{1}{\omega_{\ell}^{2}-\Omega^{2}} .
$$

Its solution defines a set of characteristic frequencies; for each of these frequencies we obtain from Eq. (9) a set of amplitudes Al, defining a normal mode of oscillation of the system.

For a beam of particles the condition that the collective force is small: compared with the focusing force is often satisfied. Then, to first order in $Z$, we can substitute $Z\left( \pm \omega_{0}\right)$ for $Z(\Omega)$ in $\mathrm{Eq}$. (10). If all particles have the same frequency, the solution of Eq. (10) is

$$
\Omega=\omega_{0}+\frac{\pi N}{\omega_{0}} \operatorname{ImZ}\left(\omega_{0}\right)-\frac{i \pi N}{\omega_{0}} \operatorname{ReZ}\left(\omega_{0}\right),
$$




$$
\Omega=-\omega_{0}-\frac{\pi N}{\omega_{0}} \operatorname{ImZ}\left(\omega_{0}\right)-\frac{i \pi N}{\omega_{0}} \operatorname{ReZ}\left(\omega_{0}\right)
$$

To obtain this result we used the property that the real and imaginary parts of $z(\omega)$ are odd and even,

$$
\begin{aligned}
& \operatorname{ReZ}(-\omega)=-\operatorname{ReZ}(\omega), \\
& \operatorname{ImZ}(-\omega)=\operatorname{ImZ}(\omega),
\end{aligned}
$$

a property that follows from the fact that $G(t)$ must be a real function.

The quantity

$$
\delta \Omega=i \pi N Z\left(\omega_{0}\right) / \omega_{0}
$$

appearing in Eq. (11) represents the shift between the collective frequency $\Omega$ and the unperturbed frequency $\omega_{0}$, and is called the coherent frequency shift.

It is interesting that solutions (11) and (12) both have the same imaginary part. Since $D s e^{-i \Omega t}$, it follows from Eq. (6) that the motion is stable if

$$
\operatorname{ReZ}\left(\omega_{0}\right)>0
$$

and unstable if

$$
\operatorname{REZ}\left(\omega_{0}\right)<0
$$

The rise time of the instability is then given by

$$
\frac{1}{\tau}=-\mathrm{N} \frac{\operatorname{Rez}\left(\omega_{0}\right)}{2 \omega_{0}}
$$

Note that for an instantaneous interaction, $G(t)=\delta(t), z(\omega)=-i$ and the system is stable. To obtain an instability there must be a real part of $z(\omega)$, producing a $\pi / 2$ phase shift between the particle oscillation and the center-ofmass oscillation, in Eq. (1).

The properties (13) and (14) of $Z(\omega)$ can be used to simplify the dispersion relation. Since, to obtain information on the stability property, it is enough to calculate the root near $\omega_{0}$, Eq. (10) can be rewritten as 


$$
1=\delta \Omega \frac{1}{N} \sum_{\ell=1}^{N} \frac{1}{\omega_{\ell}-\Omega} .
$$

For a large number of particles an integral can be substituted for the sum:

$$
1=\delta \Omega \int \mathrm{d} \omega \frac{g(\omega)}{\omega-\Omega}
$$

where $g\left(\omega_{0}\right)$ is the frequency distribution function normalized to one. To study this dispersion relation (20), let us assume that the frequency distribution is non zero only in an interval $\omega_{0}-\Delta, \omega_{0}+\Delta$ and rewrite (20) as

$$
1=\delta \Omega L(\Omega)
$$

where

$$
\begin{aligned}
L(\Omega) & =\int_{-1}^{1} \mathrm{dx} \frac{\mathrm{g}(\mathrm{x})}{\mathrm{x}-\mathrm{x}_{1}}, \\
\mathrm{x}_{1} & =\left(\Omega-\omega_{0}\right) / \Delta .
\end{aligned}
$$

For a complex impedance the solution of Eq. (20) is a complex number

$$
x_{1}=r+i \alpha
$$

obtained from the equations

$$
\frac{\delta \Omega_{R}-i \delta \Omega_{I}}{\delta \Omega_{R}^{2}+\delta \Omega_{i}^{2}}=L_{R}\left(x_{1}\right)+i L_{I}\left(x_{1}\right)
$$

where $\delta \Omega_{R}, \delta \Omega_{I}, L_{R}, L_{I}$ are the real and imaginary parts of $\delta \Omega, D_{R}\left(x_{1}\right)$ and

$$
\begin{aligned}
& L_{R}\left(x_{1}\right)=\int_{-1}^{1} \operatorname{dxg}(x) \frac{x-r}{(x-r)^{2}+\alpha^{2}}, \\
& L_{I}\left(x_{1}\right)=\int_{-1}^{1} \operatorname{dxg}(x) \frac{\alpha}{(x-r)^{2}+\alpha^{2}} .
\end{aligned}
$$


We can first solve Eq. (25) in the case where

$$
r^{2}+\alpha^{2} \gg 1
$$

which means that the collective frequency is well outside the frequency distribution $g(\omega)$. Expanding Eqs. (26) and (27) as a power series in $x /\left(r^{2}+\alpha^{2}\right)^{\frac{1}{2}}$ we obtain, to the lowest order,

$$
\begin{aligned}
\mathrm{L}_{\mathrm{R}} & =-\Delta \mathrm{r}, \\
\therefore \mathrm{L}_{\mathrm{I}} & =\Delta \alpha,
\end{aligned}
$$

and, using Eqs. (23) and (25),

$$
\Omega=\omega_{0} \cdot-\delta \Omega
$$

which is the same result as that obtained for the case in which all particles have the same frequency. In particular, if $\operatorname{ReZ}\left(\omega_{0}\right)<0$, the system is unstable.

Since $r$ and $\alpha$ are proportional to $\delta \Omega$, the condition (28) can be satisfied, for a given impedance, only for a large number of particles, $N$. If $N$ is decreased, the imaginary part of $\delta \Omega$ will decrease, and the system becomes less unstable. If $\mathrm{N}$ is decreased to the point where the condition (28) is no longer satisfied and the characteristic frequency $\Omega$ can be inside the frequency distribution, then, for a particular value of $\mathrm{N}$, a solution of the dispersion relation (25) with $\alpha \rightarrow 0^{+}$can be obtained. This solution has a rise time going to infinity and defines the maximum stable value of $\mathrm{N}$ or "threshold intensity."

In the 1 imit $\alpha \rightarrow 0^{+}$and with the relationship

$$
\lim _{\alpha \rightarrow 0^{+}} \frac{\alpha}{(x-r)^{2}+\alpha^{2}}=\pi \delta^{\circ}(x-r)
$$

the integrals (26) and (27) become

$$
\begin{gathered}
T_{{ }_{I}\left(x_{1}\right)=\pi g(r),} \\
L_{R}\left(x_{1}\right)=\lim _{\alpha \rightarrow 0+} \int_{-1}^{1} \mathrm{dxg}(x) \frac{x-r}{(x-r)^{2}+\alpha^{2}}=P \int_{-1}^{1} \mathrm{dx} \frac{g(x)}{x-r},
\end{gathered}
$$

where the last term indicates a principal value integral. 
By using Eqs. (33) and (34) Eq. (25) can now be solved for $r$ and $N$. One way to do this is to obtain $r$ from

$$
\frac{\mathrm{L}_{\mathrm{I}}(\mathrm{r})}{\mathrm{L}_{\mathrm{R}}(\mathrm{r})}=-\frac{\delta \Omega_{\mathrm{I}}}{\delta \Omega_{\mathrm{R}}}
$$

which is independent of $\mathrm{N}$, and then to obtain $\mathrm{N}$ from

$$
\mathrm{N}_{\mathrm{th}}\left|\pi \frac{2\left(\omega_{\mathrm{O}}\right)}{\omega_{\mathrm{O}}}\right|=\left\{\mathrm{L}_{\mathrm{R}}^{2}+\mathrm{L}_{\mathrm{I}}^{2}\right\}^{-\frac{1}{2}} .
$$

In Eq. (36) $\mathrm{N}$ is indicated as $\mathrm{N}_{\mathrm{th}}$, the instability threshold system intensity.

As an example let us consider the case of a parabolic frequency distribution of width $2 \Delta$,

$$
g(\omega)=\frac{3}{4 \Delta}\left\{1-\left(\frac{\omega-\omega_{0}}{\Delta}\right)^{2}\right\}
$$

which gives

$$
L(\Omega)=\frac{3}{4 \Delta} \int_{-1}^{1} d x \frac{1-x^{2}}{x-x_{1}}
$$

and for $\alpha \rightarrow 0^{+}$and $|r| \leq 1$

$$
L(\Omega)=\frac{3 \pi}{4 \Delta} F(r)
$$

with

$$
F(r)=\frac{1}{\pi}\left\{\left(1-r^{2}\right) \ln \frac{1-r}{1+r}-2 r+i \pi\left(1-r^{2}\right)\right\} .
$$

Note that when $r$ varies between -1 and +1 the quantity $|F(r)|$ changes between 0.64 and 1 (Fig. 1). The equation for the threshold intensity can be written as

$$
\frac{3 \pi}{4} \mathrm{~N}_{\text {th }} \frac{\mid \frac{}{\pi \mathrm{Z}\left(\omega_{\mathrm{o}}\right) / \omega_{\mathrm{o}}}}{\Delta|\mathrm{F}|}=1
$$

where $|F|$ is a form factor of the order of one. 
Another way to write Eq. (41) is

$$
\frac{\delta \Omega}{\Delta} \simeq 1
$$

where $\delta \Omega=\Omega-\omega_{0}$ is the coherent frequency shift that would be obtained if all the particles had the same frequency and for an intensity equal to the threshold one. This result tells us that we can have a stable system also if $\operatorname{ReZ}\left(\omega_{0}\right)<0$ if we have a frequency spread, $\Delta$, of the order of the frequency shift. This mechanism of beam stabilization is the "Landau damping."(4)

It is also important to note that for an unstable system we can modify the impedance by adding a feedback system to make $\operatorname{Re} Z\left(\omega_{0}\right) \geq 0$. Thus, we can use also a feedback stabilization system.

\section{The Vlasov Equation}

A more general formulation of the stability problem, not subject to the 1 imitations which applied to the description used in Section 2, can be obtained by using the vlasov equation. Let us consider again the system of oscillators considered in Section 2. The equation of motion (1) can be obtained from a Hamiltonian

$$
\begin{gathered}
\mathrm{H}=\mathrm{H}_{0}+\mathrm{H}_{1}, \\
\mathrm{H}_{0}=\sum_{\ell=1}^{N}\left\{\frac{1}{2} \mathrm{p}_{\ell}^{2}+\frac{1}{2} \omega_{0}^{2} \mathrm{x}_{\ell}^{2}+\mathrm{bx}_{\ell}^{4}\right\}, \\
\mathrm{H}_{1}=-\sum_{\ell=1}^{N} \mathrm{~F}_{\ell} \mathrm{x}_{\ell},
\end{gathered}
$$

where $F_{\ell}$ is the collective force, and we have added a term bx ${ }^{4}$ representing a small nonlinear force and used $p, x$ instead of $x, \dot{x}$.

The Vlasov equation uses the single-particle distribution function $t(x, p, t)$ to describe the time evolution of the system, according to the equation $(5)$

$$
\frac{\partial f}{\partial t}+\dot{x} \frac{\partial f}{\partial x}+\dot{p} \frac{\partial f}{\partial p}=0
$$

with

$$
\dot{x}=\frac{\partial H}{\partial p} \text {, }
$$




$$
\dot{p}=-\frac{\partial H}{\partial x},
$$

and $\mathrm{H}$ is now a single-particle Hamiltonian

$$
\begin{gathered}
\mathrm{H}=\mathrm{H}_{0}+\mathrm{H}_{1}, \\
\mathrm{H}_{0}=\frac{1}{2}\left(\mathrm{p}^{2}+\omega_{0}^{2} \mathrm{x}^{2}\right)+b x^{4}, \\
\mathrm{H}_{1}=-\mathrm{F}_{c} \mathrm{x} .
\end{gathered}
$$

The collective force

$$
F_{c}=N \int G\left(t-t^{\prime}\right) D\left(t^{\prime}\right) d t^{\prime}
$$

depends on $f$ itself through the definition of $D(t)$

$$
D(t)=\int x f(x, p, t) d x d p
$$

making the Vlasov equation a nonlinear equation for $f(x, p, t)$.

Equation (46) is an approximation of the Liouville equation (5) and does not describe collision processes and the irreversible approach of a system to equilibrium. It gives, however, a good description of the stability of a system.

The technique used to study the stability is to write the distribution function as a sum of an equilibrium solution and a omall perturbation

$$
f=f_{0}(x, p)+f_{1}(x, p, t)
$$

with the assumption

$$
D\left(f_{0}\right)=\int x f_{0}(x, p) d x d p \equiv 0
$$

Any function $f_{o}$ which is a function of $H_{o}$ is an equilibrium solution of the Vlasov equation, as can be seen immediately by using Eqs. (47) and (48). 
It is often convenient to use instead of the variables $x, p$ the actionangle variables $J, \psi$ obtained from the generating function

$$
\begin{gathered}
F=-\frac{1}{2} \omega_{0} x^{2} \operatorname{tg} \psi, \\
J=-\frac{\partial F}{\partial \psi}, \\
p=\frac{\partial F}{\partial x} .
\end{gathered}
$$

Frum Eqs. (57) and (58) we obtain

$$
\begin{aligned}
& x=\left(2 J / \omega_{0}\right)^{\frac{1}{2}} \cos \psi, \\
& p=-\left(2 \omega_{u} J\right)^{\frac{1}{2}} \sin \psi,
\end{aligned}
$$

and the new Hamiltonian is

$$
\overline{\mathrm{H}}=\omega_{0} \mathrm{~J}+\mathrm{b}\left(\frac{2 \mathrm{~J}}{\omega_{0}}\right)^{2} \cos ^{4} \psi-F_{c}\left(\frac{2 J}{\omega_{u}}\right)^{\frac{1}{2}} \cos \psi .
$$

If we are away from the half or quarter integer resonance, that is, if $w_{0}$ is far enough from $1 / 2$ or $1 / 4$ (modulus 1 ), we can take an average of the term proportional to b over one oscillation period and rewrite the Hamiltonian as

$$
\overline{\mathrm{H}}=\omega_{\mathrm{o}} \mathrm{J}\left\{1+\frac{3}{2} \mathrm{~b} \frac{\mathrm{J}}{\omega_{\mathrm{o}}^{3}}\right\}-\mathrm{F}_{\mathrm{c}}\left(\frac{2 \mathrm{~J}}{\omega_{\mathrm{o}}}\right)^{\frac{1}{2}} \cos \psi .
$$

Ihe term $3 b J / 2 \omega_{0}^{3}$ describes a change in frequency with amplitude due to a nonlinear (octupolar) force, since, for $F_{c}=0$,

$$
\psi^{\prime}=\frac{\partial H}{\partial J}=\omega_{o}\left\{1+3 b \frac{J}{\omega_{o}^{3}}\right\}
$$

If the Hamiltonian ( $G 2$ ) is used, the equilibrium distribution function is a function only of $\mathrm{J}, \mathrm{f}_{o}=\mathrm{f}_{\mathrm{o}}(\mathrm{J})$. The collective force is a function only of $f_{1}$, and the center-of-mass $D$ can be written as 


$$
D(t)=\int_{0}^{\infty} d J \int_{0}^{2 \pi} d \psi\left(\frac{2 J}{\omega_{0}}\right)^{\frac{1}{2}} \cos \psi f_{1}(J, \psi, t)
$$

With $\mathrm{f}_{1}$ assumed to be small, the Vlasov equation can now be written, to first order in $\mathrm{f}_{1}$, as

$$
\frac{\partial f_{1}}{\partial t}+\frac{\partial H_{o}}{\partial J} \frac{\partial f_{1}}{\partial \psi}-\frac{\partial H_{1}}{\partial \psi} \frac{\partial f_{o}}{\partial J}=0
$$

because $\partial \mathrm{f}_{0} / \partial \psi=0$. This linearized equation is adequate to describe the initial state of the instability, as long as $f_{1}$ remains a small perturbation. When, in an unstable system, $f_{1}$ grows, the full nonlinear equation should be solved to describe its evolution.

We assume now $f_{1}$ of the form

$$
f_{1}=\left\{a(J) e^{i \psi}+b(J) e^{-i \psi}\right\} e^{-i \Omega t}
$$

and substitute in Eq. (65) to obtain

$$
\begin{aligned}
& \mathrm{a}(\mathrm{J})=\frac{\mathrm{i}}{2} \mathrm{NZ}(\Omega) \overline{\mathrm{D}}\left(\frac{2 \mathrm{~J}}{\omega_{\mathrm{o}}}\right)^{\frac{1}{2}} \frac{\partial \mathrm{f}_{\mathrm{o}} / \partial \mathrm{J}}{\Omega-\partial \mathrm{H}_{\mathrm{o}} / \partial \mathrm{J}}, \\
& \mathrm{b}(\mathrm{J})=-\frac{\mathrm{i}}{2} \mathrm{NZ}(\Omega) \overline{\mathrm{D}}\left(\frac{2 \mathrm{~J}}{\omega_{0}}\right)^{\frac{1}{2}} \frac{\partial \mathrm{f}_{\mathrm{o}} / \partial \mathrm{J}}{\Omega+\partial \mathrm{H}_{\mathrm{o}} / \partial \mathrm{J}},
\end{aligned}
$$

where

$$
\bar{D}=\int d J d \psi\left(\frac{2 J}{\omega_{0}}\right)^{\frac{1}{2}} \cos \psi\left\{a(J) e^{i \psi}+b(J) e^{-i \psi}\right\}
$$

Substitution of Eqs. (67) and (68) into Eq. (69) gives the dispersion relation

$$
1=\delta \Omega \int_{0}^{\infty} \mathrm{dJ} J \frac{\partial \mathrm{f}}{\partial \mathrm{J}}\left\{\frac{1}{\Omega-\partial \mathrm{H}_{0} / \partial \mathrm{J}}-\frac{1}{\Omega+\partial \mathrm{H}_{\mathrm{o}} / \partial \mathrm{J}}\right\}
$$


As in Section 2 we need to study only the solution $\Omega \simeq \omega_{0}$ and can therefore neglect the second term in the braces in Eq. (70) and rewrite this dispersion relation in the simpler form

$$
1=\delta \Omega \int_{0}^{\infty} \mathrm{dJ} \frac{\mathrm{J}\left(\partial \mathrm{f}_{\mathrm{o}} / \partial \mathrm{J}\right)}{\Omega-\partial \mathrm{H}_{0} / \partial \mathrm{J}} .
$$

This has the same structure as Eq. (20) except that $J\left(\partial \mathrm{f}_{\mathrm{O}} / \partial \mathrm{J}\right)$ is substituted for $g(\omega)$. Changing variable from $\omega$ to $\mathrm{J}$,

$$
J=\left(\omega-\omega_{0}\right) /\left(\omega_{0}^{2} / 3 b\right)
$$

we can rewrite the integral as

$$
\int_{\omega}^{\infty} d \omega \frac{\bar{g}(\omega)}{r-\omega}
$$

wath

$$
\bar{g}(\omega)=\left(\omega_{0}^{2} / 3 b\right)^{2}\left(\omega-\omega_{0}\right)\left(\partial f_{0} / \partial J\right) J=\left(\omega-\omega_{0}\right) 3 b / \omega_{0} .
$$

All the consideration of Section 2, including the definitions of the stability limit and threshold intensity, can now be applied again.

\section{Equations of Motion for the Longitudina1. Degree of Freedom}

In this and the next sertinn we intrnduse the equations of motion for the longitudinal and transverse degrees of freedom (synchrotron and betatron motion). Th1s is done only in order to introduce our notations and define the approximations that we use. A full discussion of particle motion in an accelerator appears in E.D. Courant's lectures in this volume.

To describe the longitudinal motion of a particle we use as variables the azimuthal angle $\theta$ and the longitudinal momentum. In the approximation of neglecting the coupling to the transverse, betatron, oscillations and assuming the transverse momentum to be much smaller than the longitudinal momentum, we can use the energy as the variable associated to $\theta$.

Introducing a reference particle of energy $E_{o}$ and angle $\theta=\omega_{o} t$, we can describe any other particle in the beam by the variables $\varepsilon$, $\phi$, where

$$
\theta=\omega_{0} t+\phi(t)
$$




$$
\varepsilon=\left(E-E_{0}\right) / E_{0} \cdot
$$

It is important to note that if we work in the rotating frame defined by the variables $\phi, \epsilon$ we have to substitute $\frac{\partial}{\partial t}-\omega_{0} \frac{\partial}{\partial \phi}$ for $\frac{\partial}{\partial t}$ whenever necessary.

The equations of motion for a particle in a bunch can now be written as

$$
\begin{gathered}
\dot{\phi}=-w_{0} n \varepsilon, \\
\dot{\varepsilon}=\frac{\omega_{o}}{n} v_{s}^{2} \phi+\mu+\mu_{N L},
\end{gathered}
$$

where the "frequency slip factor" $n$ describes the change in revolution frequency with energy,

$$
\eta=-\left.\frac{1}{\omega_{0}} \frac{\partial \omega}{\partial \varepsilon}\right|_{\varepsilon=0}=\alpha-\frac{1}{\gamma^{2}}
$$

where $\alpha$ is the momentum compaction factor, $\gamma$ is the particle energy in rest mass units, and $\nu_{s}$ is the small-amplitude synchrotron tune. Equation (78) has been linearized in $\phi$ and thus takes into account only particles executing smal1amplitude synchrotron oscillations. The quantity $\mu$ describes the energy change due to interaction of the beam with its environment and goes to zero when the beam current goes to zero. The last quantity in Eq. (78), $\mu_{\mathrm{NL}}$, describes the nonlinear part of the force produced by the rf system and is included explicitly to make it possible to consider the effect of Landau damping on beam stability.

The additional energy loss describes the effect of the interaction of the beam with the environment and will be evaluated in Section 6 .

Equations (77) and (78) can be obtained from the Hamiltonian

$$
\mathrm{H}=-\frac{1}{2} \omega_{\mathrm{o}} \eta \varepsilon^{2}-\frac{1}{2} \frac{\omega_{\mathrm{o}}}{\eta} v_{\mathrm{s}}^{2} \phi^{2}+\mathrm{U}+\mathrm{U}_{\mathrm{NL}}
$$

with

$$
\begin{gathered}
\mu=-\frac{\partial U}{\partial \phi}, \\
\mu_{N L}=-\frac{\partial U_{N L}}{\partial \phi} .
\end{gathered}
$$


For a beam with many bunches we can generalize Eqs. (77) and (78), introducing bunch coordinates $\phi_{p}$, $\varepsilon_{p}$, with $p$ being the bunch label. Tf the position of the center of bunch $\mathrm{p}$ is $\Phi_{\mathrm{p}}$, the bunch coordinate $\phi_{\mathrm{p}}$ is related to $\phi$ by $\phi_{\mathrm{P}}=\phi-\Phi_{\mathrm{p}}$. For B equally spaced bunches $\Phi_{\mathrm{p}}=2 \pi \mathrm{p} / \mathrm{B}, \mathrm{p}=0,1, \ldots, \mathrm{B}-1$.

Using bunch coordinates we can describe each bunch with a different distribution function $f(p)\left(\phi_{p}, \varepsilon_{p}, t\right)$. For a beam with $B$ bunches we write the equations of synchrotron oscillations as

$$
\begin{gathered}
\dot{\phi}_{\mathrm{p}}=-n \omega_{\mathrm{o}} \varepsilon_{\mathrm{p}}, \\
\dot{\varepsilon}_{\mu}=\frac{\omega_{0} \nu_{s}^{2}}{11} \phi_{l}+\mu_{\mathrm{p}}+\mu_{\mathrm{NV}}, \quad p=0,1, \ldots, \mathrm{B}-1,
\end{gathered}
$$

and the Hamiltonian as

$$
H=-\sum_{n=0}^{B-1} \frac{1}{2} \omega_{0} n \varepsilon_{n}^{2}+\frac{1}{2} \frac{\omega_{0} v^{2} s}{n} \phi_{n}^{2}+U+U_{N L}
$$

where

$$
\mu_{p}=-\frac{\partial U}{\partial \phi_{p}}
$$

function

We can introduce action-angle variables $\mathrm{J}, \zeta$ by using the generating

$$
G=-\frac{1}{2} \frac{\nu_{s}}{|\eta|} \sum_{n=0}^{B-1} \phi_{n}^{2} \operatorname{tg} \zeta_{n}
$$

which gives

$$
\begin{aligned}
& \phi_{n}=\left(\frac{2|n| J_{n}}{\nu_{s}}\right)^{\frac{1}{2}} \cos \zeta_{n}, \\
& \varepsilon_{n}=-\left(\frac{2 \nu_{s} J_{n}}{|\eta|}\right)^{\frac{1}{2}} \sin \zeta_{n} .
\end{aligned}
$$


With these variables the Hamiltonian is

$$
\overline{\mathrm{H}}=-\frac{\eta}{|\eta|} v_{\mathrm{s}} \omega_{\mathrm{o}} \sum_{\mathrm{n}=0}^{\mathrm{B}-\mathrm{l}} \mathrm{J}_{\mathrm{n}}+\overline{\mathrm{U}}+\overline{\mathrm{U}}_{\mathrm{NL}}
$$

where $\bar{U}$ is a function of $J_{n}, \zeta_{n}$ and $\bar{U}_{\text {NL }}$ is averaged over the $\zeta^{\prime} s$ to obtain a function of the $J_{n}$ only.

5. Equations of Motion for the Transverse Oscillations

We consider particles oscillating in one transverse direction, neglecting the coupling between the two betatron oscillation modes. We also simplify the problem by neglecting the AG structure of the machine and using the smooth approximation to describe the betatron motion. This last approximation is rather good, and its use can be justified by the analysis of the general theory. (6)

If $\mathrm{y}$ is the transverse direction of oscillation, we can write the equation of motion in the form

$$
\ddot{y}+\nu_{o}^{2} \omega_{o}^{2}(1+2 P \varepsilon) y=F(\phi, t)+N(y)
$$

where $F(\phi, t)$ is the collective self force, $N(y)$ is the nonlinear force producing a change in frequency with oscillation amplitude, and the term $2 \mathrm{P} \varepsilon$ describes the change in frequency due to a variation in energy. The quantity $P$ is given by

$$
P=\xi-\eta
$$

with $\eta$ given by Eq. (79) and the "chromaticity" $\xi$ defined as

$$
\xi=\frac{1}{v_{0}} \frac{d \nu_{0}}{d \varepsilon}
$$

which describes the change in tune with energy.

The longitudinal variables $\varepsilon, \phi$ satisfy the equations of synchrotron motion (77) and (78). However, in these synchrotron equations we neglect both the collective term $\mu$, and the nonlinear term $\mu_{\mathrm{NL}}$, so that the longicudinal muliun in $\varepsilon, \phi$ is that of a harmonic oscillator. 
The equation of motion (91) can be derived from the Hamiltonian

$$
\mathrm{H}=\mathrm{H}_{\mathrm{OT}}+\mathrm{H}_{\mathrm{OL}}+\mathrm{H}_{\mathrm{NL}}-\mathrm{F}(\phi, \mathrm{t}) \mathrm{y}
$$

with

$$
\begin{aligned}
& \mathrm{H}_{\mathrm{OT}}=\frac{1}{2} \dot{\mathrm{y}}^{2}+\frac{1}{2} \nu_{\mathrm{O}}^{2} \omega_{\mathrm{O}}^{2}(1+2 \mathrm{P} \varepsilon) \mathrm{y}^{2}, \\
& \mathrm{H}_{\mathrm{OL}}=-\frac{1}{2} \omega_{\mathrm{O}} \eta \varepsilon^{2}-\frac{1}{2} \frac{\omega_{\mathrm{O}} \nu_{\mathrm{s}}^{2}}{\eta} \phi^{2},
\end{aligned}
$$

and $\mathrm{H}_{\mathrm{NL}}$ describing the nonlinear fonre. Serond-order tormc in $y$ arc ncglected in the equation for $\phi$. function

We now perform a canonical transformation defined by the generating

$$
\theta=-\frac{1}{2} \nu_{0} \omega_{0} y^{2} L g \psi
$$

to introduce the transverse action-angle variables $I, \psi$ :

$$
\begin{gathered}
y=\left(\frac{2 I}{\nu_{0} \omega_{0}}\right)^{\frac{1}{2}} \cos \psi, \\
\dot{y}=-\left(2 \nu_{0} \omega_{0} I\right)^{3 / 2} \dot{s} \dot{L} \Psi .
\end{gathered}
$$

With these variables the Hamiltonian is

$$
\overline{\mathrm{H}}=\nu_{\mathrm{O}} \omega_{\mathrm{O}} \mathrm{I}(1+\mathrm{P \varepsilon}(1+\cos 2 \psi))+\mathrm{H}_{\mathrm{OL}}-\mathrm{F}(\phi, t)\left(\frac{2 \mathrm{I}}{\nu_{\mathrm{o}} \omega_{\mathrm{o}}}\right)^{\frac{1}{2}} \cos \psi+\mathrm{H}_{\mathrm{NL}} .
$$

The term $\nu_{0} \omega_{0} I P \varepsilon \cos 2 \psi$ is a fast oscillating term, except for the case when $\nu_{0}$ $\simeq 1 / 2$, and can be neglected. We also take an average of $\overline{\mathrm{H}}_{\mathrm{NL}}$ over $\psi$, assuming ${ }^{\circ}$ that nonlinear resonances can be neglected, and

$$
\left\langle\overline{\mathrm{H}}_{\mathrm{NI}}>=\nu_{n} \omega_{n} \mathrm{D}(\mathrm{I})\right.
$$


For a bunched beam, of $B$ bunches, it is convenient to introduce "bunch coordinates" $y_{K}, \phi_{K}$ and write the transverse equation of motion as

$$
\ddot{y}_{K}+v_{0}^{2} \omega_{0}^{2}\left(1+2 P \varepsilon_{K}\right) y_{K}=F\left(\phi_{K}, t\right)+N\left(y_{K}\right)
$$

The notation is the same as that used in Eq. (91). The equations for $\varepsilon_{\mathrm{K}}$, $\phi_{\mathrm{K}}$ are given by Eqs. (83) and (84) with the nonlinear and the collective forces taken equal to zero.

The equations of motion (102), (83), and (84) can be obtained from a Hamiltonian

$$
H=\sum_{K=0}^{B-1}\left(H_{O T, K}+H_{O L, K}+H_{C, K}+\bar{H}_{N L, K}\right)
$$

where

$$
\begin{gathered}
H_{\mathrm{OT}, \mathrm{K}}=\frac{1}{2} \mathrm{y}_{\mathrm{K}}^{2}+\frac{1}{2} \nu_{\mathrm{O}}^{2} \omega_{\mathrm{O}}^{2}\left(1+2 \mathrm{P} \varepsilon_{\mathrm{K}}\right) \mathrm{y}_{\mathrm{K}}^{2}, \\
\mathrm{H}_{\mathrm{OL}, \mathrm{K}}=-\frac{1}{2} \frac{\omega_{\mathrm{O}} \nu_{\mathrm{s}}^{2}}{n} \phi_{\mathrm{K}}^{2}-\frac{\omega_{\mathrm{O}}{ }^{n}}{2} \varepsilon_{\mathrm{K}}^{2}, \\
\mathrm{H}_{\mathrm{C}, \mathrm{K}}=-\mathrm{F}\left(\phi_{\mathrm{K}}, t\right) \mathrm{y}_{\mathrm{K}},
\end{gathered}
$$

and we neglect second-order terms in the equation of motion for $\phi$. As in the previous case we now introduce action-angle variables $I_{K}$, $\psi_{K}$ for the transverse motion and $J_{K}, \zeta_{K}$ for the longitudinal motion. Using the generating function (97) for the transverse variables, we have

$$
\begin{gathered}
y_{K}=\left(\frac{2 I_{K}}{\nu_{0} \omega_{0}}\right)^{\frac{1}{2}} \cos \psi_{K}, \\
\dot{y}_{K}=-\left(2 \nu_{0} \omega_{0} I_{K}\right)^{\frac{1}{2}} \sin \psi_{K},
\end{gathered}
$$

and $\phi_{K}, \varepsilon_{K}$ are given by Eqs. (88) and (89). 
With these variables we obtain the Hamiltonian

$$
\begin{aligned}
\bar{H} & =\sum_{K=0}^{B-1}\left\{\nu_{0} \omega_{0}\left(1+P \varepsilon_{K}\right) I_{K}+\nu_{o} \omega_{O} D\left(I_{K}\right)\right. \\
& \left.-\frac{\eta}{T \eta} \nu_{s} \omega_{0} J_{K}-F\left(J_{K}, \zeta_{K}, t\right)\left(\frac{2 I_{K}}{\nu_{0} \omega_{0}}\right)^{\frac{1}{2}} \cos \psi_{K}\right\},
\end{aligned}
$$

having used Eq. (101) to write the nonlinear part.

6. The Transverse and Longitudinal Collective Forces

\subsection{The charge and current density}

We want to evaluate the force produced by the beam on a test particle, $\ell$. To do this we describe the beam charge and current density with the help of the. single-particle distribution function, $f$. We use a coordinate system $x, y, \theta$ (or $x, y, s=R \theta, R$ being the average accelerator radius) and use as conjugate variables, momenta, the quantity $\dot{x}, \dot{y}, \varepsilon$.

We write the distribution function as

$$
F=\frac{N}{R} f(x, y, \theta ; \dot{x}, \dot{y}, \varepsilon ; t)
$$

where $N$ is the number of particles in the beam, $R$ is introduced because we use the variable $\theta$ instead of $s$ and $f(x, y, \theta ; \dot{x}, \dot{y}, \varepsilon ; t)$ is normalized to one.

We assume that the two transverse betatron oscillation modes are uncoupled and consider the case in which the beam particles are oscillating only in one direction, say $y$. The charge density can now be defined in terms of the distribution function as

$$
e \rho(x, y, \theta ; t)=\frac{e N}{R} \int f(x, y, \theta ; \dot{x}, \dot{y}, \varepsilon ; t) d \dot{x} d \dot{y} d \varepsilon
$$

The transverse current density is given by

$$
J_{x}(x, y, \theta ; t)=\frac{e N}{R} \int \dot{x} f(x, y, \theta ; \dot{x}, \dot{y}, \varepsilon ; t) d \dot{x} d \dot{y} d \varepsilon
$$




$$
J_{y}(x, y, \theta ; t)=\frac{e N}{R} \int \dot{y} f(x, y, \theta ; \dot{x}, \dot{y}, \varepsilon ; t) d \dot{x} d \dot{y} d \varepsilon
$$

For the case of one-dimensional oscillations in the $y$ direction we assume

$$
\mathrm{J}_{\mathbf{x}} \equiv 0
$$

The longitudinal velocity, $\dot{s}=\dot{\theta} R$, is the sum of a constant term, $v$, the velocity of the reference particle, plus a small term proportional to $\varepsilon$. We assume the beam to be relativistic, $v \simeq c$, and neglect the small term, as compared with $\mathrm{v}$, in evaluating the longitudinal current density. With this approximation we have

$$
J_{s}=e v \rho
$$

To further simplify the problem we also assume that there is no coupling between the transverse and longitudinal collective modes of oscillation and consider the two cases separately. We consider first the fields and forces produced by a bunch and afterward extend the results to a beam with many bunches.

For the transverse oscillation modes we assume that the distribution function is of the form

$$
f=\frac{N}{R}\left\{f_{o}(x, y, \phi ; \dot{x}, \dot{y}, \varepsilon)+f_{l}(x, y, \phi ; \dot{x}, \dot{y}, \varepsilon) e^{-i w w_{o} t}\right\}
$$

where $\mathrm{N}$ is the number of particles in the bunch and $\nu$ the coherent oscillation tune. The term $f_{o}$ in Eq. (116) represents an equilibrium solution. The term $f_{l}$ is a small perturbation.

We also make the following assumptions:

1) For the equilibrium term the density can be factorized in a longitudinal and a transverse term

$$
\rho_{0}(x, y, \theta ; t)=\frac{N}{R} \int f_{0} d \dot{x} d \dot{y} d \varepsilon=\frac{N}{R} \lambda(\phi) h_{0}(x, y)
$$

with $h_{0}(x, y)$ and $\lambda(\phi)$ normalized to one:

2) The equilibrium term has zero dipole moment: 


$$
\int \mathrm{yf}_{\mathrm{o}}(\mathrm{x}, \dot{\mathrm{x}}, \mathrm{y}, \dot{\mathrm{y}}, \phi, \varepsilon) \mathrm{dxd} \dot{\mathrm{x}} \mathrm{dy} \dot{\mathrm{y}} \mathrm{d} \varepsilon=0
$$

3) The perturbation term must satisfy the normalization condition

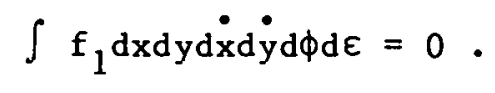

write

4) Both $f_{o}$ and $f_{1}$ are periodic functions of $\phi=\theta-\omega_{0} t$; in particular we

$$
\lambda(\phi)=\frac{1}{2 \pi I_{n}} \sum_{p} I_{p} e^{i p \phi}
$$

where

$$
\therefore-\mu N v / 7 \pi R
$$

is the average bunch current and the $I_{p}$ are the Fourier components of the bunch current; for the perturbed density

$$
\rho_{1}(x, y, \phi)=\int f_{1} \dot{d} \dot{x} d \dot{y} d \varepsilon
$$

we write

$$
\rho_{1}(x, y, \phi)=\sum_{p} h_{p}(x, y) e^{i p \phi}
$$

and for the transverse current

$$
\begin{aligned}
& J_{y, 1}(x, y, \phi)=\int \dot{y f}_{1} d \dot{x} d y d t, \\
& J_{y, 1}(x, y, \phi)=\sum_{p} b_{p}(x, y) e^{i p \phi} .
\end{aligned}
$$

From the equation of continuity of current we obtain the relationship

$$
i v \omega_{o} h_{p}(x, y)=\frac{\partial}{\partial y} b_{p}(x, y)
$$


The charge and current density can then be written as

$$
\begin{gathered}
e \rho=\frac{e N}{R} h_{o}(x, y) \sum_{p} \frac{I_{p}}{2 \pi I_{o}} e^{i p \phi}+\frac{e N}{R} \sum_{p} h_{p}(x, y) e^{i p \phi-i v \omega_{o} t}, \\
J_{y}=\frac{e N}{R} \sum_{p} b_{p}(x, y) e^{i p \phi-i w_{o} t} .
\end{gathered}
$$

To describe the case of longitudinal oscillations we assume

$$
f=\frac{N}{R} g_{o}(\phi, \varepsilon) h_{o}(x, y, \dot{x}, \dot{y})+\frac{N}{R} g_{1}(\phi, \varepsilon) h_{1}(x, y, \dot{x}, \dot{y}) e^{-i \Omega \omega_{o} t}
$$

where $\Omega$ is the collective frequency for a longitudinal oscillation mode. Since we are interested only in longitudinal effects, we assume that the transverse currents are zero, $\mathrm{J}_{\mathrm{x}}=\mathrm{J}_{\mathrm{y}}=0$.

The charge and longitudinal current density are now given by

$$
\begin{gathered}
e \rho=\frac{e N}{R} n_{0}(\phi) d_{0}(x, y)+\frac{e N}{R} n_{1}(\phi) d_{1}(x, y) e^{-i \omega_{0} \Omega t}, \\
J_{s}=e v \rho,
\end{gathered}
$$

where the $d(x, y)$ and $n(\psi)$ are obtained by integrating Eq. (129) over $\dot{x}, \dot{y}, \varepsilon$. The terms $n_{o}(\phi), d_{o}(x, y)$ are normalized to one,

$$
\int \mathrm{n}_{\mathrm{o}}(\phi) \mathrm{d} \phi=1
$$

and for the perturbation term

$$
\int \mathrm{n}_{1}(\phi) \mathrm{d} \phi=0
$$




\subsection{The transverse force}

The electric and magnetic field generated by the beam can be obtained from Maxwel1 equations, using $\rho$ and $\mathrm{J}_{\mathrm{y}}$, given by Eqs. (127) and (128), as sources. Using the potential and vector potential in the Lorentz gauge, we have

$$
\begin{aligned}
& V=-4 \pi e \rho, \\
& \underline{A}=-\frac{4 \pi}{c} \underline{J} .
\end{aligned}
$$

To solve these equations we assume

$$
\begin{gathered}
V=\frac{e N}{R} \int_{p} v_{p}(x, y) e^{i p \phi-i v \omega_{0} t}, \\
A_{s}=0, \\
A_{y}=\frac{e N}{R c} \sum_{p} A_{p}(x, y) e^{i p \phi-i v \omega_{o} t}, \\
A_{s}=B V,
\end{gathered}
$$

where $\beta=v / c$. We note that the charge density (127) has two terms and the first can be obtained from the second by assuming $\nu=0, h_{p}=h_{0} I_{p}$. Now we solve Eq. (132) considering only the second term as a source. At the end we make a linear combination of the two solutions.

The scalar and vector potentials are obtained by solving the two equations

$$
\begin{aligned}
& \left\{\frac{\partial^{2}}{\partial x^{2}}+\frac{\partial^{2}}{\partial y^{2}}+\lambda_{p}^{2}\right\} v_{p}(x, y)=-4 \pi h_{p}(x, y), \\
& \left\{\frac{\partial^{2}}{\partial x^{2}}+\frac{\partial^{2}}{\partial y^{2}}+\lambda_{p}^{2}\right\}_{p}(x, y)=-4 \pi b_{p}(x, y),
\end{aligned}
$$

with the proper boundary conditions on the walls of the vacuum chamber. The quantity $\lambda_{\mathrm{p}}$ is 


$$
\lambda_{p}^{2}=\frac{\omega_{o}^{2}}{c^{2}}\left((p+v)^{2}-\frac{p^{2}}{\beta^{2}}\right)
$$

The time component of the field oscillates at the frequency $(\nu+p) \omega_{0}$, $\nu$ being the collective frequency and $p \omega_{0}$ describing the effect of the accelerator periodicity.

The transverse force is given by

$$
F=e E_{y}+e B H x
$$

and using Eqs. (136) and (137) we can write this as

$$
F=\frac{e^{2} N}{R} \sum_{p} c_{p}(x, y) e^{i p \phi-i w_{o} t}
$$

with

$$
c_{p}=-\frac{1}{\gamma^{2}} \frac{\partial v_{p}(x, y)}{\partial y}+i v \frac{\omega_{o}}{c} A_{p}(x, y) .
$$

The Lorentz condition .

$$
\frac{1}{c} \frac{\partial V}{\partial t}+\operatorname{div} \underline{A}=0
$$

gives us the relationship

$$
\text { iv } \frac{(1)}{c} v_{p}(x, y)=\frac{\partial A_{p}(x, y)}{\partial y} \text {. }
$$

If we introduce the Green functions $G_{p}$, $H_{p}$, satisfying the equations -

$$
\begin{aligned}
& \left\{\frac{\partial^{2}}{\partial x^{2}}+\frac{\partial^{2}}{\partial y^{2}}+\lambda_{p}^{2}\right\}_{p}\left(x-x^{\prime}, y-y^{\prime}\right)=-4 \pi \delta\left(x-x^{\prime}\right) \delta\left(y-y^{\prime}\right), \\
& \left\{\frac{\partial^{2}}{\partial x^{2}}+\frac{\partial^{2}}{\partial y^{2}}+\lambda_{p}^{2}\right\}_{p}\left(x-x^{\prime}, y-y^{\prime}\right)=-4 \pi \delta\left(x-x^{\prime}\right) \delta\left(y-y^{\prime}\right),
\end{aligned}
$$


and the proper boundary conditions, then the solution of Eqs. (140) and (141) can be expressed as

$$
\begin{aligned}
& V_{p}(x, y)=\int G_{p}\left(x-x^{\prime}, y-y^{\prime}\right) h_{p}\left(x^{\prime}, y^{\prime}\right) d x^{\prime} d y^{\prime}, \\
& A_{p}(x, y)=\int H_{p}\left(x-x^{\prime}, y-y^{\prime}\right) b_{p}\left(x^{\prime}, y^{\prime}\right) d x^{\prime} d y^{\prime} .
\end{aligned}
$$

Furthermore, the Green functions can be written as the sum of the two terms:

$$
\begin{aligned}
& G_{p}=G_{p}^{V}+G_{p}^{W}, \\
& H_{p}=H_{p}^{V}+H_{p}^{W} .
\end{aligned}
$$

The terms $G_{\mathrm{P}}^{\mathrm{V}}$, $\mathrm{H}_{\mathrm{P}}^{\mathrm{V}}$ are the Green functions, satisfying the outgoing boundary conditions, for a beam in vacuum. The wall terms, $G_{p}^{W}$, $H_{p}^{W}$ are added so that the boundary conditions on the walls can be satisfied.

The fields and forces obtained from Eqs. (140) and (141) are nonlinear. However, to study the stability problem for the, dipole mode of instability it is enough to consider the linear part only.

For the field in vacuum the Green functions $G_{p}, H_{p}$ are given by $(7)$

$$
G_{p}\left(x-x^{\prime}, y-y^{\prime}\right)=H_{p}\left(x-x^{\prime}, y-y^{\prime}\right)=i \pi H_{0}^{(1)}\left(\lambda_{p} P\right)
$$

with

$$
P^{2}=\left(x-x^{\prime}\right)^{2}+\left(y-y^{\prime}\right)^{2}
$$

The field corresponding to the equilibrium distribution can be calculated assuming $b_{p}=0, h_{p}=\left(I_{p} h_{o}\right) / 2 \pi I_{o}$. For a uniform cylindrical beam of radius a one has

$$
\frac{\partial V_{P}}{\partial y}=-\frac{i I_{P}}{a I_{0}} H_{l}\left(\lambda_{p} a\right) J_{1}\left(\lambda_{p} r\right)(y / r)
$$

with $\mathrm{r}^{2}=\mathrm{x}^{2}+\mathrm{y}^{2}$. For wavelength long compared with the beam size, $\lambda_{\mathrm{p}} \mathrm{a} \ll 1$, one has 


$$
\frac{\partial V_{p}}{\partial y} \simeq-\frac{I_{P}}{\pi I_{o} a^{2}} y
$$

In the case of a more complicated beam geometry one has to substitute an "effective beam transverse area" for the quantity $\pi a^{2}$. The field produced by the perturbation is obtained, in the long wavelength limit, by displacing the beam by a distance equal to the dipole moment. In this approximation we have

$$
\frac{\partial V_{p}}{\partial y}=\frac{2 I p}{I_{0} a^{2}} D_{p}
$$

with the dipole moment, $D_{p}$, given by

$$
D_{P}=\int y h_{P}(x, y) d x d y
$$

In the same approximation we also have $A_{p}=0$.

For the part of the fields due to the walls we can expand the Green functions in a power series to obtain

$$
\begin{aligned}
\frac{\partial V_{p}^{W}(x, y)}{\partial y} & =\frac{\partial G_{p}^{W}(0,0)}{\partial y} \int h_{p}\left(x^{\prime}, y^{\prime}\right) d x^{\prime} d y^{\prime} \\
& +\frac{\partial^{2} G_{p}^{W}(0,0)}{d y^{2}} \int\left(y-y^{\prime}\right) h_{p}\left(x^{\prime}, y^{\prime}\right) d x^{\prime} d y^{\prime}+\ldots .
\end{aligned}
$$

and similarly for $H$. The first term on the right-hand side is a constant and can be neglected. For the second term we have two different results according. to whether we consider the equilibrium density $h_{o}$ or the perturbation $h_{p}$. For the equilibrium case, using Eq. (118), we have

$$
\frac{\partial V_{p}^{(0)}}{\partial y}=y \frac{I_{p}}{2 \pi I_{0}} \frac{\partial^{2} G_{p}^{W}(0,0)}{\partial y^{2}}
$$

For the perturbation we have, using Eq. (159),

$$
\frac{\partial V_{p}(x, y)}{\partial y}=-\frac{\partial^{2} G_{p}^{w}(0,0)}{\partial y^{2}} D_{p} \text {. }
$$


We can perform a similar expansion for $A_{p}$. In this case there is a contribution only from the perturbation, which, using Eq. (126), we can write as

$$
A_{p}(x, y) \simeq-i \vee \omega_{o} H_{p}(0,0) D_{p}
$$

Collecting the terms (157) and (161), proportional to y, and using Eqs. (144) and (145), we obtain what is called the incoherent force:

$$
F_{\text {inc }}=\frac{2 e y}{\gamma^{2} \beta c} \sum_{p} I_{p}\left\{\frac{1}{a^{2}}-\frac{1}{2} \frac{\partial^{2} G_{p}^{W}(0,0)}{\partial y^{2}}\right\} e^{i p \phi}
$$

This force produces a change in the focusing force, which can be introduced in the equations of motion by substituting $v_{0}-\delta v_{\text {inc }}$ for $v_{0}$, with

$$
\delta v_{i n c}=-\frac{F_{i n c} c^{2}}{2 y E_{n} v_{n} w_{n}^{2}}
$$

Introducing the incoherent impedance

$$
z_{p}^{i n c}=-i \frac{R}{e I_{p}^{y B}} \int_{o}^{2 \pi} F e^{-i p \phi} d \phi
$$

we have from Eq. (164)

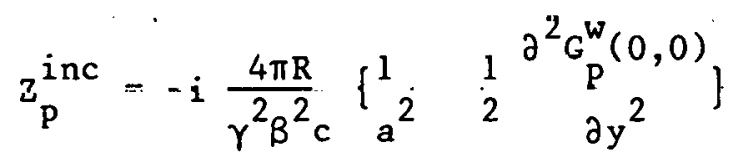

in $c \cdot g . s$. units or

$$
z_{P}^{\text {inc }}=-i \frac{R z_{o}}{\gamma^{2} R}\left\{\frac{1}{a^{2}}-\frac{1}{2} \frac{\partial^{2} G_{P}^{W}}{\partial y^{2}}\right\}
$$

in M.K.S. units, $Z_{o}=377 \Omega$ being the vacuum impedance. Using the impedance we can rewrite the incoherent force as

$$
F_{\text {inc }}=\frac{i e \beta y}{2 \pi R} \sum_{p} I_{p} z_{p}^{i n c} e^{i p \phi}
$$




$$
\delta \nu_{\text {inc }}=-\frac{\text { ieR }}{4 \pi \nu_{o} \beta E} \sum_{\mathrm{p}} I_{p} z_{p}^{i n c} e^{i p \phi} .
$$

The force produced by the perturbation can be obtained from Eqs. (158), $(162),(163),(144)$, and $(145)$ as

$$
F=-\frac{e^{2} N}{R} \sum_{P} D_{p}\left\{\frac{2}{\gamma^{2} a^{2}}-\frac{1}{\gamma^{2}} \frac{\partial^{2} G_{p}^{w}}{\partial y^{2}}-\frac{\nu^{2} \omega_{o}^{2}}{c} H_{p}\right\} e^{i p \phi-i v \omega_{o} t} .
$$

Introducing a transverse impedance $z_{1}$ defined as

$$
Z_{\perp}(p+\nu)=-i \frac{R \int_{0}^{2 \pi} F e^{-i p \phi+i \nu \omega_{0} t} d \phi}{e I_{0}^{\beta D} p},
$$

giving the ratio of the total force per revolution to the transverse current $I_{o} D_{p}$ at the frequency $(\nu+p) \omega_{o}$, we can rewrite Eq. (171) as

$$
F=\frac{i e I_{o} \beta}{2 \pi R} \sum_{p} D_{p} z_{1}(p+\nu) e^{i p \phi-i \nu \omega_{o} t}
$$

with

$$
Z_{J}(p+\nu)=\frac{i 4 \pi}{c} \frac{2 \pi R}{B^{2}} \cdot\left\{\frac{1}{\gamma^{2} a^{2}}-\frac{1}{2 \gamma^{2}} \frac{\partial^{2} G_{p}^{W}}{\partial y^{2}}-\frac{\nu^{2} \omega_{o}^{2}}{2 c} H_{p}^{W}\right\}
$$

The transverse impedance is an impedance per unit length. The expression (17.4) is in c.g.s. units. To obtain it in M.K.S. units we can substitute for $4 \pi / c$ the vacuum impedance, $Z_{0}=377 \Omega$.

Note that the $Z_{\perp p}$ defined by Eq. (174) satisfies the symmetry conditions (13) and (14).

Let us consider now the case of a bunched beam with B equally spaced bunches. The distribution function for bunch $K$ and the force produced by bunch $\mathrm{K}$ are obtained from Eqs. (115) and (173) by shifting the coordinate $\phi$ by $2 \pi \mathrm{K} / \mathrm{B}$, 
with $K=0,1, \ldots, B-1$. The total force acting on bunch $\ell$ can then be written as a sum over the contribution from all bunches, evaluated at $\phi_{\ell}=\phi-2 \pi l / B$,

$$
F^{(\ell)}=\frac{i e I_{o} \beta}{2 \pi R} \sum_{K=0}^{B-1} \sum_{p} D_{p}^{(K)} z_{1}(p+v) e^{i p \phi_{\ell}-2 \pi i p \frac{K-\ell}{B}-i \omega_{o} t} .
$$

The dipole moment for bunch $\mathrm{K}$ is defined, with use of the distribution function

$$
\begin{aligned}
f^{(K)} & =\frac{N}{R}\left\{f_{u}^{(K)}\left(x, y, \phi-2 \pi \frac{K}{B} ; \dot{x}, \dot{y}, \varepsilon\right)\right. \\
& \left.+t_{1}^{(K)}\left(x, y, \psi-2 \pi \frac{K}{B} ; \dot{x}, \dot{y}, \varepsilon\right) e^{-i\left(w_{0} t\right.}\right\},
\end{aligned}
$$

as

$$
D_{p}^{(K)}=\frac{1}{2 \pi} \int y f_{1}^{(K)}(x, y, \phi ; \dot{x}, \dot{y}, \varepsilon) e^{-i p \phi} d x d y d \phi d \dot{x} d \dot{y} d \varepsilon
$$

In writing Eq. (177) we have also assumed that all bunches have the same number of particles, N. The current $I_{0}$, in Eq. (175), is the average current per bunch.

\subsection{The longitudinal force}

To find the potential and the vector potential we again use Eqs. (134) and (135) with the charge and current densities given by Eqs. (130) and (131).

Since we have $J_{x}=J_{y}=0, J_{s}=\beta_{c} \rho$, we can also assume that $A_{x}=A_{y}=0$ and $A_{s}=B V$. We are then left with a single equation for the scalar potential V.

By using the perturbed charge density as a source, the equation for $V$ can be easily obtained. The case of the equilibrium density can then be obtained for $\Omega=0$, with proper label changes. The longitudinal density must be periodic in $\phi$ so that we can write

$$
e \rho=\frac{e N}{R} d_{1}\left(x ; y \sum_{p} \Lambda_{p} e^{i p \phi-i \Omega \omega_{o} t}\right.
$$

with 


$$
\begin{aligned}
\Lambda_{\mathrm{P}} & =\frac{1}{2 \pi} \int \mathrm{n}_{1}(\phi) \mathrm{e}^{-i p \phi} \mathrm{d} \phi \\
& =\frac{1}{2 \pi} \int \mathrm{d} \phi \mathrm{d} \dot{\varepsilon} g_{1}(\phi, \varepsilon) \mathrm{e}^{-i \mathrm{p} \phi} .
\end{aligned}
$$

We now as sume

$$
V=\frac{e N}{R} \sum_{p} \Lambda_{p} V_{p}(x, y) e^{i p \phi-i \Omega \omega_{o} t}
$$

and obtain from Eq. (134) the equation

$$
\left\{\frac{\partial^{2}}{\partial x^{2}}+\frac{\partial^{2}}{\partial y^{2}}+\lambda_{p}^{2}\right\} v_{p}(x, y)=-4 \pi d_{1}(x, y)
$$

that must be solved with the proper boundary conditions. The quantity $\lambda_{p}$ in Eq. (181) is

$$
\lambda_{\mathrm{p}}^{2}=\left(\omega_{\mathrm{o}} / \mathrm{c}\right)^{2}\left\{(\Omega+\mathrm{p})^{2}-\mathrm{p}^{2} / \mathrm{B}^{2}\right\}
$$

To evaluate the energy loss that appears in the synchrotron oscillation equation, Eq. (78), the electric field has to be calculated first.

$$
E_{s}=-i \frac{2 \pi \beta I}{R c} \sum_{p} \Lambda_{p} v_{p}(x, y)\left\{\frac{p}{\gamma^{2} \beta^{2}}-\Omega\right\} e^{i p \phi-i \Omega \omega_{o} t}
$$

We assume that to evaluate the energy loss we can use the field on axis and that $\phi$ does not change during a revolution. With this approximation we obtain

$$
\mu=-i e \frac{4 \pi}{c} \frac{B \omega_{o} I_{o}}{2 E_{o}} \sum_{p} \Lambda_{p} v_{p}(0,0)\left\{\frac{p}{\gamma^{2} \beta^{2}}-\Omega\right\} e^{i p \phi-i \Omega \omega_{o} t} .
$$

As in the previous section we now introduce a longitudinal impedance

$$
z_{1 i}(p+\Omega)=\frac{4 \pi}{c} \frac{B}{2} v_{p}(0,0)\left\{\frac{p}{\gamma^{2} B^{2}}-\Omega\right\}
$$

where $z_{0}$ can be substituted for $4 \pi / c$ if we use M.K.S. units. 
The collective frequency $\Omega$ is a small term and is zero in the absence of a perturbation. Since we will use the impedance to obtain a linearized Vlasov equation, we can neglect the $\Omega$ term in Eq. (185).

Introducing the impedance in Eq. (184) we obtain

$$
\mu=-i e \frac{\omega_{o} I_{0}}{E_{0}} \sum_{p} \Lambda_{p} z_{11}(p+\Omega) e^{i p \phi-i \Omega \omega_{o} t}
$$

Another quantity of interest is the "potential" $U$ defined by Eq. (81). This is written as

$$
U=e \frac{\omega_{0} I_{0}}{E_{0}} \sum_{p} \frac{Z_{11}(p+\Omega)}{p} \Lambda_{p} e^{i p \phi-i \Omega \omega_{0} t}
$$

For a beam with B bunches we can write Eq. (178) as

$$
\rho=\frac{e N}{R} d_{1}(x, y) \sum_{K=0}^{B-1} \sum_{p} \Lambda_{p}^{(K)} e^{i p\left(\phi+2 \pi \frac{K}{B}\right)-i \Omega \omega_{o} t}
$$

with

$$
\Lambda_{\mathrm{p}}^{(\mathrm{K})}=\frac{1}{2 \pi} \int \mathrm{d} \phi \mathrm{d} \varepsilon \mathrm{g}_{1}^{(K)}(\phi, \varepsilon) \mathrm{e}^{-i p \phi}
$$

and obtain the energy loss of bunch $\ell$ from

$$
U^{(l)}=\frac{e \omega_{o} I_{0}}{E_{0}} \sum_{K=0}^{B-1} \sum_{p} \frac{z_{l l}(p+\Omega)}{p} \Lambda_{p}^{(k)} e^{i p \phi_{l}+i 2 \pi p(k-l) / B-i \Omega \omega_{0} t} .
$$

\section{The Model Impedance}

In Section 6 we have written the collective forces acting on the beam in terms of a longitudinal and a transverse impedance. For the space-charge terms, however, this impedance characterizes the beam environment, which comprises the vacuum chamber and all the radio-frequency cavities, pickup electrodes, bellows, and other devices needed to build and operate the accelerator. In this situation, calculation of the impedance and of the field and forces can be difficult. 
From the experimental point of view it is possible to measure the impedance of each part of the vacuum system during construction of the ring and thus to evaluate the total ring impedance. Information on the ring impedance can also be obtained from measurements of the beam properties as a function of the beam current.

These measurements, (8) performed on several accelerators, show that one can often use a simple model in which the impedance is described as a combination of a few terms. The first is the impedance of a straight vacuum pipe with a uniform cross section, made of a low resistivity metal like stainless steel or aluminum. The effect of the radio-frequency system is described by a few lowor medium-frequency resonances, at $\omega<\mathrm{c} / \mathrm{b}, \mathrm{b}$ being the vacuum chamber radius. A final term is used to describe the effect of all other parts, which can introduce many high frequency resonances, which are all averaged to obtain the "broad-band impedance."(8)

The impedance of a resistive cylindrical pipe of radius $b$ has been derived in many papers. (9) In the long wavelength approximation, $\omega<\mathrm{c} / \mathrm{b}$ or $\lambda \mathrm{b}<1$, it can be written, neglecting a term proportional to $\Omega$ as discussed in Section 6.3 , in the form

$$
z_{11}(\omega) \simeq z_{o}\left\{\frac{B g_{o}}{2 \beta^{2} \gamma^{2}} p+\frac{R}{b} \mathscr{R}(\omega)\right\}
$$

where the first term describes the space-charge effect, which, for a uniform cylindrical beam of radius a, is given by

$$
g_{0}=1+2 \ln (b / a)
$$

and the second term describes the effect of the wall resistivity with the wall impedance $\mathscr{R}(\omega)$ given by

$$
\mathscr{R}(\omega)=(1-i) \frac{\omega}{2 c} \delta(\omega)
$$

where $\delta(\omega)$ is the skin depth for a wall of conductivity $\sigma$ :

$$
\therefore \delta(\omega)=c /(2 \pi \omega \sigma)^{\frac{1}{2}} \text {. }
$$

The first term in Eq. (191), describing the space-charge effect, is a capacitive impedance; the second term, due to the resistivity, has a capacitive and a resistive term. 
In the same approximation the transverse impedance is given by (9)

$$
Z_{1}(\omega)=i Z_{0} R\left\{\frac{1}{B^{2} \gamma^{2}}\left(\frac{1}{a^{2}}-\frac{1}{b^{2}}\right)-(1+i) \frac{\delta(\omega)}{b^{3}}\right\}
$$

where again the first term describes the space-charge effect and the second the effect of the resistivity.

The broad-band impedance is that of a parallel $\mathrm{R}, \mathrm{C}, \mathrm{L}$ circuit resonating at a frequency of the order of $c / b$ and with a $Q$ of the order of one. ( 8 ) The value of the shunt impedance, $R_{O}$, completely defines this impedance and characterizes an accelerator.

Assuming that the $r f$ resonances can also be described by an equivalent $R$, L, C parallel circuit, we can obtain the total ring model impedance by adding to the longitudinal impedance (191) a number of terms

$$
Z_{11}(\omega)=i R_{0} \frac{\omega}{\omega_{R}} \frac{\left(\omega / \omega_{R}\right)-i Q\left(1-\left(\omega / \omega_{R}\right)^{2}\right)}{\left(\omega / \omega_{R}\right)^{2}+Q^{2}\left(1-\left(\omega / \omega_{R}\right)^{2}\right)^{2}}
$$

where $\omega_{R}$ is the resonance frequency. To describe the resonant rf cavity impedance we use in Eq. (196) the rf cavity parameters. To describe the broad-band impedance we assume $\omega_{R} \sim c / b$ and $Q \simeq 1$.

For the transverse impedance associated with the same structures we assume that all resonant transverse modes of the rf cavity are damped to avoid strong beam instabilities. With the broad-band impedance we associate a transverse broad-band impedance $(10)$

$$
z_{1}(\omega)=2 \frac{k}{d^{2}} \frac{7_{11}((1))}{\left(\omega / \omega_{0}\right)}
$$

We now use the results of the previous discussion to define a longitudinal and a transverse "model impedance." We also make the following additional assumptions:

1) All resonant, high Q, impedanees are eliminated by a prupés system design; if this is not the case they must be taken into account, and they can produce very strong beam instabilities.

2) In the longitudinal impedance we neglect the space-charge and image term, which is usually small and can be neglected in comparison with the resistive wall or broad-band impedance term; this assumption is violated for low $\gamma^{\prime} s(\gamma \sim 1)$. 
3) We use for the transverse broad-band impedance and the resistive wall impedance the relationship (197).

4) We use the condition $\lambda_{a} \ll 1$ to simplify the resistive pipe impedance.

5) We also use $\lambda b \ll 1$ to simplify the same impedance; this is justified if for $\lambda b \geqslant 1$ the resistive pipe impedance is smaller than the broad-band impedance, since in this case we can use a simplified expression for a small term even if the approximation on this small term is not good.

Using all these assumptions we can now write (using the M.K.S. system)

$$
z_{11}(\omega)=-z_{0} \frac{R}{b} \frac{1-i}{2} \frac{\omega}{c} \delta(\omega)+z_{11}^{B}(\omega)
$$

where $z_{11}^{B}(\omega)$ is the broad-band impedance defined by Eq. (196), and

$$
z_{1}(\omega)=i Z_{0} R\left\{\frac{1}{\beta^{2} \gamma^{2}}\left(\frac{1}{a^{2}}-\frac{1}{b^{2}}\right)-(1+i) \frac{\delta(\omega)}{b^{3}}-\frac{2 i}{d^{2}} \frac{z_{11}^{B}(\omega) / z_{o}}{\left(\omega / \omega_{0}\right)}\right\}
$$

These two expressions define the model impedances, which can be used in many beam stability calculations to obtain at least an order of magnitude est imate.

\section{Longitudinal Instabilities of a Coasting Beam}

We start the study of longitudinal instabilities by considering the simplest case, a uniform coasting beam. This case was the first to be studied theoretically, (11) and the predicted instability, called the negative mass instability, was later observed in several accelerators.

For a coasting beam there is no radio-frequency system so that we can take $\nu_{s}=0$ and $\mu_{\mathrm{NL}}=0$ in the equations of motion (77) and (78). We assume that initially the longitudinal density distribution function is uniform. Following Eq. (129) and neglecting the transverse coordinates, we assume

$$
f(\phi, \varepsilon, t)=\frac{N}{R}\left\{g_{0}(\varepsilon)+g_{1}(\phi, \varepsilon) e^{-i \Omega \omega_{0} t}\right\}
$$

We can also use the periodicity in $\phi$ to write 


$$
g_{1}(\phi, \varepsilon)=\sum_{p} g_{p}(\varepsilon) e^{i p \phi}
$$

The coefficients $g_{p}(\varepsilon)$ are related to the $\Lambda_{p}$ introduced in Section 6 , Eq. (179), by

$$
\Lambda_{p}=\int d \varepsilon g_{p}(\varepsilon)
$$

The Hamiltonian for our system can be obtained from Eqs: (80) and (187):

$$
H=-\frac{1}{2} n \omega_{0} \varepsilon^{2}+\frac{e I_{0} \omega_{0}}{E_{0}} \sum_{p} \frac{z_{11}(\Omega+p)}{p} \Lambda_{p} e^{i p \phi-i \Omega \omega_{0} t} .
$$

With this Hamiltonian the linearized Vlasov equation is

$$
-i \omega_{0} \sum_{p}^{i}(\Omega+p \eta \bar{\varepsilon}) g_{p} e^{i p \phi-i \Omega(1)} u^{\dagger}=\frac{i e \omega_{n} I_{n}}{E_{0}} \frac{\partial g_{n}}{\partial \varepsilon} \sum_{p} L_{11}(p+\Omega) \Lambda_{p} e^{i p \phi-i \Omega t}
$$

giving

$$
g_{p}(\varepsilon)=-\frac{e I_{o} Z_{11}(p+\Omega)}{E_{o}} \Lambda_{p} \cdot \frac{\partial g_{o} / \partial \varepsilon}{\Omega+p \eta \varepsilon}
$$

Integrating over $\varepsilon$ we also have the dispersion relation

$$
1=-\frac{e I_{o} Z_{11}(p+\Omega)}{E_{o}} \int d \varepsilon \frac{\partial g_{o} / \partial \varepsilon}{\Omega+p \eta \varepsilon}
$$

This equation defines the eigenvalues $\Omega$ for the beam normal mode $e^{i p \phi}$.

Introducing the quantities

$$
\begin{gathered}
\mathrm{x}_{\mathrm{p}}=-\frac{j l}{\mathrm{p} \eta}, \\
\mathrm{L}\left(\mathrm{x}_{\mathrm{p}}\right)=\int \mathrm{d} \varepsilon \frac{\partial \mathrm{g}_{\mathrm{o}}}{\partial \varepsilon} \frac{1}{\varepsilon-\mathrm{x}_{\mathrm{p}}},
\end{gathered}
$$


and

$$
\delta \Omega=-\frac{e I_{0}}{\eta E_{0}} \frac{Z_{11}(p+\Omega)}{p},
$$

we can rewrite Eq. (206) as

$$
1=\delta \Omega L\left(x_{p}\right)
$$

To study the dispersion relation (210) let us introduce explicitly the real and imaginary part of $x_{p}$,

$$
x_{p}=r+i \alpha
$$

and also of $L\left(x_{p}\right)$ and $\delta \Omega$

$$
\begin{gathered}
L\left(x_{P}\right)=L_{R}+i L_{I}, \\
\delta \Omega=X+i Y .
\end{gathered}
$$

The dispersion relation (210) can now be written as

$$
\frac{X-i Y}{X^{2}+Y^{2}}=L_{R}+i L_{I}
$$

with

$$
\begin{aligned}
& \mathrm{L}_{\mathrm{R}}=\int \mathrm{d} \varepsilon \frac{\varepsilon-\mathrm{r}}{(\varepsilon-r)^{2}+\alpha^{2}} \frac{\partial \mathrm{g}_{\mathrm{o}}}{\mathrm{d} \varepsilon}, \\
& \mathrm{L}_{\mathrm{I}}=\int \mathrm{d} \varepsilon \frac{\varepsilon-\mathrm{r}}{(\varepsilon-r)^{2}+\alpha^{2}} \frac{\partial \mathrm{g}_{\mathrm{o}}}{\mathrm{d} \varepsilon} .
\end{aligned}
$$

8.1. The negative mass instability

We now want to study these equations in two cases of incerest:

1) beam with zero energy spread, and

2) the threshold current for a beam with non-zero energy spread. 
For the first case let us assume

$$
g_{0}(\varepsilon)=\delta(\varepsilon)
$$

From Eqs. (215), (216), and (217) we obtain

$$
\begin{aligned}
& L_{R}=\frac{r^{2}-\alpha^{2}}{\left(r^{2}+\alpha^{2}\right)^{2}}, \\
& L_{I}=\frac{2 \alpha r}{\left(r^{2}+\alpha^{2}\right)^{2}},
\end{aligned}
$$

and, combining these two equations,

$$
L_{R}+i L_{I}=\frac{1}{(1-i \alpha)^{2}}
$$

Equation (229) together with (223) gives

$$
(x-i \alpha)^{2}=(X+\dot{Y} Y)
$$

In the case of a perfectly conducting cylindrical vacuum chamber we have, from Eys. (209) alud (191),

$$
x=-\frac{e I}{\eta E} \frac{Z_{o} g_{o}}{2 B \gamma^{2}}, \quad y=0
$$

and the solutions are,

$$
\begin{gathered}
\text { if } \quad n<0, \\
r=|x|^{\frac{1}{2}}, \quad u=0,
\end{gathered}
$$

which means that $\Omega$ is real and the beam is stable;

$$
\text { if } n>0 \text {, }
$$




$$
r=0, \quad \alpha=|x|^{\frac{1}{2}}
$$

and the beam is unstable.

The frequency slip factor is given by. Eq. (79) as $\eta=\alpha-1 / \gamma^{2}$, or, with the transition energy $\gamma_{\mathrm{T}}=\alpha^{-\frac{1}{2}}$, as

$$
\eta=\frac{1}{\gamma_{T}^{2}}-\frac{1}{\gamma^{2}}
$$

With this definition,

$$
\begin{array}{lll}
n>0 & \text { if } & \gamma>\gamma_{T}, \\
n<0 & \text { if } & \gamma<\gamma_{T} .
\end{array}
$$

Hence we can say that a beam in a perfectly conducting vacuum chamber is unstable for $n>0$, i.e., above transition energy. This instability is called the "negative mass instability" and was the first beam instability to be discovered.

The mechanism producing the negative mass instability is the following. For a beam with uniform longitudinal density there is no longitudinal electric field and no force acting on the particles. Let us create a local perturbation, a bump, in the longitudinal density. This perturbation will produce a spacecharge longitudinal electric force $\mathrm{eE}_{\phi} \simeq-e \partial \lambda / \partial \phi$, acting in the direction away from the bump. This force will increase the energy of the particles in front of the bump and decrease the energy for particles in back of it. In the negative mass regime the front particle will decrease its revolution frequency $\omega$ and the back particle will increase $\omega$, so that both particles will move toward the bump and increase its magnitude. In other words any local small increase in the density will grow, thus producing in the end a non-uniform particle distribution.

Using Eqs. (226) and (207) we can predict the initial growth rate of the instability:

$$
\frac{1}{\tau}=p \omega_{0}\left\{\frac{e I_{o} z_{0}}{2 E_{0}|\eta|}\right\}_{o}^{\frac{1}{2}} \text {. }
$$

On the other hand the linearized theory that we have developed cannot give us any information on the beam final state. That would require use of the full nonlinear Vlasov equation and is outside the scope of this work. Solution of the nonlinear problem is very difficult, and most of the work done has been based on numerical calculations. 


\subsection{The effect of the energy spread and the threshold current}

In the general case in which the beam has a non-zero energy spread, we can obtain the eigenvalues, $x_{p}$, of our problem from Eqs. (214), (215), and (216).

Following the discussion of Section 1 we can look for solutions such that $\alpha \rightarrow 0^{+}$, defining a limit value for the stable current, or a threshold current.

We consider only the situation in which the energy is larger than the transition energy, $\eta>0$, so that the beam is unstable if $\alpha>0$.

In the 1 imit $\alpha \rightarrow 0^{+}$, with use of the relationship

$$
\lim _{\alpha \rightarrow 0^{+}} \frac{I}{(\varepsilon-r)^{2}+\alpha^{2}}=\pi \delta(\varepsilon-r) .
$$

the integral (216) becomes

$$
\mathrm{L}_{\mathrm{I}}=\left.\pi \frac{\partial \mathrm{g}_{\mathrm{o}}}{\partial \varepsilon}\right|_{\varepsilon=\mathrm{r}}
$$

and '(215) becomes

$$
\mathrm{L}_{\mathrm{R}}=\lim _{\alpha \rightarrow 0^{+}} \int \mathrm{d} \varepsilon \frac{\varepsilon-\mathrm{R}}{(\varepsilon-r)^{2}+\alpha^{2}} \frac{\partial \mathrm{g}_{\mathrm{o}}}{\partial \varepsilon} .
$$

The dispersion relation (214) can now be solved for $r$ and. $I_{0}$. The value of $I_{0}$ defines the threshold intensity, $I_{t h}$. One way to solve Eq. (215) is to use the equation

$$
\therefore \quad \frac{\mathrm{L}_{\mathrm{I}}}{\mathrm{L}_{\mathrm{B}}}=-\frac{\mathrm{Y}}{\mathrm{X}}
$$

to obtain $r$, and for this value of $r$ to evaluate the threshold current, $I_{t h}$, from

$$
\frac{e I_{0, t h} \frac{Z_{11}(p)}{p_{p}}|| L \mid}{E_{0} \eta}=1
$$

where the impedance has been evaluated, to first order, for $\Omega=0$.

Lèt us evaluate explicitly the case in which the energy distribution is parabolic: 


$$
g_{0}(\varepsilon)=\frac{3}{4 \sqrt{5} \sigma_{\varepsilon}}\left\{1-\left(\frac{\varepsilon}{\sqrt{5} \sigma_{\varepsilon}}\right)^{2}\right\}
$$

$\sigma_{\varepsilon}$ being the r.m.s. value of $\varepsilon$. Using Eqs. (230), (231), and (234), we obtain for the case

$$
\left|\frac{\mathrm{R}}{\sqrt{5} \sigma_{\varepsilon}}\right| \leq 1
$$

that

$$
\begin{gathered}
\mathrm{L}_{\mathrm{I}}=-\frac{3 \pi}{2} \frac{\mathrm{r}}{\left(\sqrt{5} \sigma_{\varepsilon}\right)^{3}}, \\
\mathrm{~L}_{\mathrm{R}}=\frac{2}{\pi} \mathrm{L}_{\mathrm{I}} \mathrm{G}(\mathrm{r}),
\end{gathered}
$$

with

$$
G(r)=\frac{\sqrt{5} \sigma_{\varepsilon}}{r}+\frac{1}{2} \ln \left|\frac{\sqrt{5} \sigma_{\varepsilon}-r}{\sqrt{5} \sigma_{\varepsilon}+r}\right| .
$$

For the case $\left|r / \sqrt{5} \sigma_{\varepsilon}\right|>1$ one has $L_{I}=0$ and it is possible to have a solution with $\alpha \rightarrow 0^{+}$only if $\mathrm{Y}=0$.

Equation (232) for $r$ can now be written as

$$
\frac{2}{\pi} G(r)=-\frac{X}{Y}
$$

Note that $G(-r)=-G(r)$.

In Figure 2 are plotted $G(\bar{R})$ and $\bar{F}=(5 / 3) \sigma_{\varepsilon}^{2}|L|$ versus $\bar{R}=r /\left(\sqrt{5} \sigma_{\varepsilon}\right)$. This figure shows that whatever is the value of $r$ obtained by solving Eq. (239), the value of $|L|$ is nearly constant and $|L| \simeq 1 / \sigma_{\varepsilon}^{2}$.

A similar result can be obtained for all reasonably well behaved distributions. Using this result we can write

$$
|L|=1 /\left(F \sigma_{\varepsilon}^{2}\right)
$$

where $F$ is a form factor, of the order of one, depending on the shape of the 
energy distribution and on the ratio, $\mathrm{X} / \mathrm{Y}$, of the real to the imaginary part of the impedance.

With the help of Eq. (240) we can rewrite the expression (233) for the threshold current in the convenient form(12)

$$
\frac{\mathrm{eI}_{o, t h} \frac{\mathrm{Z}_{\mathrm{p}}}{\mathrm{p}} \mid}{\mathrm{E}_{\mathrm{o}} n F \sigma_{\varepsilon}^{2}}=1 \text {. }
$$

Note that, for a given impedance, the threshold current increases as the square of the r.m.s. energy spread. The reason for this effect is that particles with different energies circulate with different angular velocities, thus reducing the coherence properties of the beam.

\section{Longitudinal Instabilities of Bunched Beams}

\subsection{General considerations and equations of motion}

The analysis of the longitudinal instabilities of bunched beams is more complicated than that of coasting beams. Although one can write the general equations describing the beam dynamics and the stability properties, it is not possible to obtain a general solution of these equations for a realistic beam charge distribution and wall impedance. It is, however, possible to obtain solutions valid in certain regions of the parameter space of this problem.

Let us introduce a time scale using the characteristic times of the beam and of its environment: the bunch duration, $\mathrm{L} / \mathrm{C}$; the revolution time, To; the period of the perturbing electromagnetic field, $\mathrm{T}_{\mathrm{p}}$; the synchrotron oscillation period, $T_{s}$; and the instability rise time, $\tau_{i}$. We always assume $\tau_{i}>T_{0}$.

We can consider two cases:

I) slow instabilities, with

$$
\tau_{i}>T_{s}>T_{0}
$$

II) fast instabilities, with

$$
\mathrm{T}_{s}>\bar{\tau}_{i}>\mathrm{T}_{0} \cdot
$$

Cases I and II can be divided in two subcases:

a) long wavelength, with

$$
\frac{\mathrm{L}}{\mathrm{CT}} \ll 1
$$


b) short wavelength, with

$$
\frac{L}{c T_{P}} \gg 1
$$

For each of these cases it is possible to write an approximate expression for the stability condition.

Case Ia, the coupled bunch instability, will be discussed in Section 9.3; case Ib, the high frequency slow instability, in Section 9.4; and case IIb, the microwave instability or bunch lengthening effect, in Section 9.5.

\subsection{Sllow instabilities}

To study this problem we use the variables J, $\zeta$ introduced in section 4 . We consider a beam with $B$ equal bunches, equally spaced, and write the distribution function for bunch $K$ as

$$
f^{(K)}=\frac{N}{R}\left\{g_{0}(J)+g_{1}^{(K)}(J, \zeta) e^{-i \Omega \omega_{0} t}\right\}
$$

Using Eqs. (90) and (190) we can write the Hamiltonian for bunch $\ell$ as

$\bar{H}^{(\ell)}=-\nu_{s} \omega_{0} J_{\ell}+\frac{e \omega_{0} I_{0}}{E_{0}} \sum_{K=0}^{B-1} \sum_{p} \Lambda_{p}^{(K)} \frac{z_{11}(p+\Omega)}{p} e^{i p\left(\phi_{\ell}+2 \pi(K-l) / B\right)-i \Omega \omega_{0} t}+U_{N L}$

where $\phi_{l}$ is a function of $\mathrm{J}_{\ell}, \zeta_{l}$ and for simplicity we have assumed the energy to be above the transition energy, $\eta>0$. The quantity $\Lambda_{\mathrm{p}}^{(\mathrm{K})}$ is given by Eq. (189).

We now expand $e^{i p \phi}$ as a Fourier series in $\zeta$ to obtain

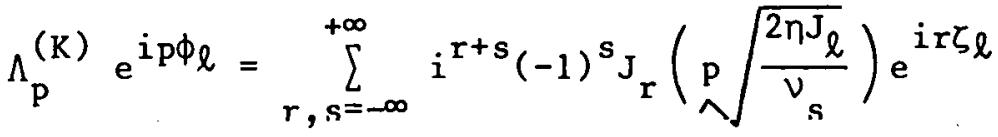

$$
\begin{aligned}
& \times \frac{1}{2 \pi} \int \mathrm{dJd} \zeta g_{1}^{(K)}(J, \zeta) J_{s}\left(\sqrt[p]{\frac{2 \pi J}{\nu_{s}}}\right) e^{i s \zeta}
\end{aligned}
$$


For the case of slow instabilities we can take an average over a synchrotron period and keep only the slowly oscillating terms $r+s=0$. Then Eq. (248) can be simplified to

$$
\begin{aligned}
& \Lambda_{p}^{(K)} e^{i p \phi l} \simeq \sum_{r=-\infty}^{+\infty} J_{r}(\underbrace{\frac{2 n J \ell}{v_{s}}}_{s}) e^{i r \zeta \ell} \\
& \times \frac{1}{2 \pi} \int d J d \zeta g_{1}^{(K)}(J, \zeta) J_{r}\left(\sqrt[p]{\frac{2 n J}{v_{s}}}\right) e^{-i r \zeta} .
\end{aligned}
$$

Finally the Hamiltonian (247) can be approximated, by using the expression (249) $\operatorname{Lor} \Lambda_{\mathrm{p}}^{(\mathrm{C})}$.

If we also make the assumption

$$
g_{1}^{(K)}(J, \zeta)=\underset{g_{q}}{\mathfrak{n}(K)}(J) e^{i q \zeta},
$$

then the expression (249) becomes

$$
\Lambda_{p}^{(K)} e^{i p \phi_{l}}=J_{q}\left(\underset{\Lambda}{\frac{2 n J_{\ell}}{v_{B}}}\right) e^{i q \zeta_{\ell}} \sigma_{\varphi}^{(K)}(p)
$$

with

$$
\sigma_{q}^{(K)}(p)=\int_{0}^{\infty} \mathrm{dJ}_{g_{q}^{\prime}}^{r(K)}(J) J_{q}\left(\underset{\sim}{\frac{2 \eta J}{v_{s}}}\right) .
$$

We will see that Eq. (250) is an eigenfunction in $\zeta$ of the Vlasov equation and represents a state in which all particles have the same phase q $\zeta$.

To write the Vlasov equation we now assume that the only effect of the U term produced by the equilibrium distribution is to modify the focusing force, i.e., to change $v_{s}$. For large currents this modification can be large, and the shape of the potential well in which the particles oscillate can be modified, (9) producing a change in bunch length. Here we will not discuss this effect in detail and assume that it produces a change in $\nu_{s}$ described by the nonlinear potential U $\mathrm{U}_{\mathrm{NL}}$. 
We can now write the linearized Vlasov equation as

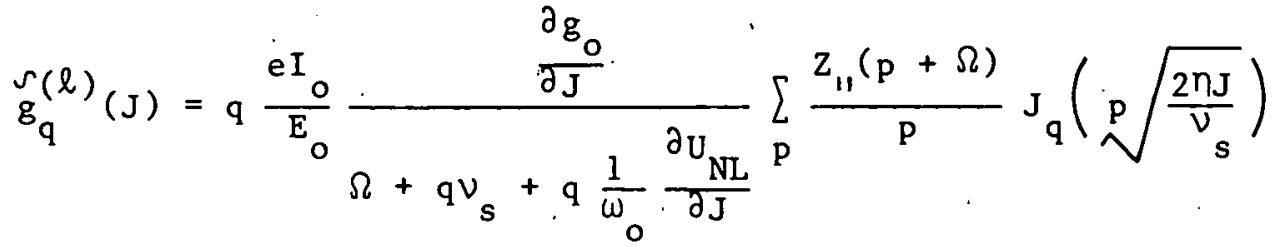

$$
\begin{aligned}
& \times \sum_{K=0}^{B-1} \cdot \sigma_{q}^{(K)}(p) e^{2 \pi i p(K-\ell) / B} .
\end{aligned}
$$

It is interesting that with the assumption $(250), g^{(K)}(J, \zeta) \simeq e^{i q \zeta}$, we have obtained an equation, (253), which is independent of $\zeta$ and in which the different $q$ modes are not coupled. We will call these modes the "synchrotron modes" and will refer to the index $p$ as the index of "azimuthal modes."

Considering Eq. (253) as a matrix equation for the vector $\tilde{g}_{\mathrm{q}}^{\sim}(\ell)$, $\ell=0, \ldots, B-1$, one can see that this matrix is a cyclic matrix in $\ell, K$ and that its solutions are of the form

$$
\stackrel{\tilde{g}}{q}_{\mathrm{q}}^{(l)}(\mathrm{J})=\mathrm{A}_{\mathrm{q}, \mathrm{s}}(\mathrm{J}) \mathrm{e}^{2 \pi \mathrm{is} \ell / \mathrm{B}}, \quad \mathrm{s}=0, \ldots, \mathrm{B}-1 .
$$

The index s defines the coupled bunch modes. For a given $s$ the bunches oscillate with a phase difference $2 \pi \mathrm{s} / \mathrm{B}$. With use of Eq. (254), Eq. (253) becomes

$$
\begin{aligned}
A_{q, 8}(J) & =\frac{e_{0}^{B}}{E_{0}} \frac{\frac{\partial g_{0}}{\partial J}}{\Omega+q\left(\nu_{s}+\frac{1}{\omega_{0}} \frac{\partial U_{N L}}{\partial J}\right)} \\
& \times \sum_{h} \frac{Z_{11}(h B-s+\Omega)}{h B-s} J_{q}\left((h B-s) \sqrt{\left.\frac{2 \pi J}{\nu_{s}}\right) \bar{\sigma}_{q, s}(h B-s)}\right.
\end{aligned}
$$


with

$$
\bar{\sigma}_{q, s}(h B-s)=\int_{0}^{\infty} d J A_{q, s}(J) J_{q}\left((h B-s) \sqrt{\frac{2 \eta J}{v_{s}}}\right),
$$

equations already derived by several authors. (13)

Our problem is now reduced to finding the eigenvalues and eigenvectors of Eq. (255). We have no general solutions of these equations, although particular solutions have been found for certain forms of the impedance, or by introducing other approximations. In the next sections we will discuss some of these solutions.

\subsection{Coupled bunch longitudinal instabilities}

Using the conditions (242) and (244) we can simplify the integral equation. (255) by expanding the Bessel functions, for small values of the argument, as $J_{q}(z) \simeq(z / 2) q / q !$. Examination of the simplified equations shows that we can obtain a solution of the form (14)

$$
A_{q, s}(J) \simeq \frac{\frac{\partial g_{0}}{\partial J}}{\Omega+q\left(\nu_{s}+\frac{1}{\omega_{0}} \frac{\partial U_{N L}}{\partial J}\right)}\left(\frac{\eta J}{2 \nu_{s}}\right)^{q / 2} .
$$

The corresponding eigenvalue is a solution of the dispersion integral

$$
\begin{gathered}
l=\frac{{ }^{e I}{ }_{0}^{B}}{\left(q \frac{1}{1}\right) ! E_{0}} \sum_{h} z_{11}(h B-s+\Omega)(h B-s)^{q-1} \int_{0}^{\infty} d J \frac{\frac{\partial g_{u}}{\partial J}\left(\frac{n J}{2 \nu_{s}}\right)^{q / 2}}{\Omega+q\left(\nu_{s}+\frac{\partial U_{N L}}{\partial J}\right)} \\
\times J_{q}\left((h B-s) \sqrt{\frac{2 n J}{v_{s}}}\right) .
\end{gathered}
$$

In the absence of Landau damping, $\partial \mathrm{U}_{\mathrm{NL}} / \partial \mathrm{J}=0$, and for a Gaussian bunch of r.m.s. length $\sigma_{\phi}$, 


$$
g_{0}(J)=\frac{\eta}{2 \pi \nu_{s} \sigma_{\phi}^{2}} e^{-\eta J / \nu_{s} \sigma_{\phi}^{2}}
$$

we obtain

$$
\Omega+q \nu_{s}=-\frac{e I_{o} B \eta \sigma_{\phi}^{2 q-2}}{2 \pi \nu_{s} E_{o}{ }^{2}(q-1) !} z_{\text {eff }}^{(q)}
$$

with

$$
z_{e f f}^{q}=\sum_{h} z_{11}\left(h B-s-q \nu_{s}\right)(h B-s)^{2 q-1} e^{-\sigma_{\phi}^{2}(h B-s)^{2} \cdot / 2} .
$$

The effective impedance has been evaluated, to zero order, for $\Omega=-q \nu_{s}$.

These results were obtained by several authors (15) to explain the first observations of coupled bunch instabilities and have been widely discussed in the literature.

The eigenvalues (260) are characterized by two numbers, $s, q$. The number $q$ gives the oscillation mode: dipole for $q=1$, quadrupole for $q=2$, etc.; the number $s$ gives the phase shift, $2 \pi s / B$, between bunches. The impedance $Z_{11}(\omega)$ has to be evaluated at a multiple of the bunch revolution frequency, $h B \omega_{0}$, minus $s \omega_{0}$ plus the q-th side band of the synchrotron frequency. (16)

In the absence of Landau damping the stability condition is given by $\operatorname{Im} \Omega \leq 0$, which, for $\eta>0$, with use of the symmetry properties (14), can also be written as

$$
\begin{aligned}
& -s^{2 q-1} e^{-\frac{1}{2} \sigma_{\phi}^{2} s^{2}} \operatorname{ImZ_{11}}\left(s+q \nu_{s}\right) \\
& +\sum_{h>0}\left\{(h B-s)^{2 q-1} e^{-\frac{1}{2} \sigma_{\phi}^{2}(h B-s)^{2}} \operatorname{ImZ}_{11}\left(h B-s-q \nu_{s}\right)\right. \\
& \left.-(h B+s)^{2 q-1} e^{-\frac{1}{2} \sigma_{\phi}^{2}(h B+s)^{2}} \operatorname{ImZ}_{11}\left(h B+s+q \nu_{s}\right)\right\} \geq 0 .
\end{aligned}
$$

For a perfectly conducting vacuum pipe $\operatorname{ImZ_{11}}=0$ and the beam is stable. 
For $q=1, s=0$ the impedance is often dominated by the resonance at the accelerating frequency $h^{*} \mathrm{~B} \omega_{0}$. In this case the stability condition becomes

$$
h^{*} B e^{-\frac{1}{2} \sigma_{\phi}{ }^{2}\left(h^{*} B\right)^{2}}\left\{\operatorname{ImZ}_{11}\left(h^{*} B-\nu_{s}\right)-\operatorname{ImZ}_{11}\left(h^{*} B+\nu_{s}\right)\right\} \geq 0
$$

so that the impedance at the lower side-band must be larger than that at the higher side-band. This stability condition was first introduced by Robinson.(17)

\subsection{The high frequency slow instability}

In this section we consider again the slow instabilities, described by the integral equation (255), without using the low frequency approximation (244). The high frequencies violating Eq. (244) can influence the internal bunch motion, and to study this effect it is sufficient to consider a single bunch, for which case $s=0$.

Neglecting the bunch mode index, we can rewrite Eq. (255) as

$$
\begin{gathered}
A_{q}(J)=\frac{q e I_{0}}{E_{o}} \frac{\frac{\partial g_{o}}{\partial J}}{\Omega+q \nu_{s}\left(1+\frac{1}{\omega_{o} \nu_{s}} \frac{\partial U_{N L}}{\partial J}\right)} \\
\quad \times \sum_{p} \frac{z_{11}(p+\Omega)}{p} J_{q}\left(p / \frac{2 \eta J}{\nu_{s}}\right) \bar{\sigma}_{q}(p)
\end{gathered}
$$

with

$$
\bar{\sigma}_{q}(p)=\int_{0}^{\infty} d_{\mathcal{q}}(J) J_{q}\left(\sqrt[p]{\frac{2 \eta J}{v_{s}}}\right) .
$$

To further simplify the discussion we assume $\partial \mathrm{U}_{\mathrm{NL}} / \partial \mathrm{J}=0$, thus neglecting Landau damping. Equation (264) has been obtained and studied by Sacherer. 
Let us introduce a new function

$$
\mu_{q}(J)=\left(\frac{\partial g_{o}}{\partial j}\right)^{-\frac{1}{2}} A_{q}(J)
$$

and rewrite Eq. (264) explicitly as an integral equation

$$
\mu_{q}(J)=\frac{q e I}{E_{o}\left(\Omega+q \nu_{s}\right)} \int_{0}^{\infty} d J^{\prime} \mu_{q}\left(J^{\prime}\right) G\left(J^{\prime} J^{\prime}\right)
$$

with

$$
\begin{aligned}
G\left(J, J^{\prime}\right) & =\left\{\frac{\partial g_{o}(J)}{\partial J} \frac{\partial g_{0}\left(J^{\prime}\right)}{\partial J^{\prime}}\right\}^{\frac{1}{2}} \sum_{p=1}^{\infty} J_{q}(\underbrace{p}_{\frac{p}{v_{s}}} \cdot J_{q}(\underbrace{}_{\frac{p}{v_{s}}}) \\
& \times \frac{z_{11}\left(p-q v_{s}\right)+z_{11}^{*}\left(p+q \nu_{s}\right)}{p}
\end{aligned}
$$

where we have evaluated the impedance to zero order for $\Omega=-q \nu_{s}$ and used the symmetry properties (13) and (14).

The Green function $G\left(J, J^{\prime}\right)$ is symetric. If the quantity $Z_{11}\left(p-q v_{s}\right)+$ $\mathrm{Z}_{11}^{*}\left(\mathrm{p}+\mathrm{q} \nu_{\mathrm{s}}\right)$ is real, the eigenvalues of Eq. (268) are also real and the beam is stable. This is, for instance, the case for a perfectly conducting wall, or for a constant imaginary part of the impedance.

Let us introduce a complete orthonormal set of eigenfunctions, $F_{m}^{q}(J)$, of the integral equation (267), and let us write

$$
\begin{gathered}
\mu_{q}(J)=\sum_{m} D_{q, m} F_{m}^{q}(J), \\
\Lambda_{m, p}^{q}=\int_{0}^{\infty} d J\left(\frac{\partial g}{\partial J}\right)^{1 / 2} J_{q}\left(\sqrt[p]{\frac{2 \pi J}{\nu_{s}}}\right) F_{m}^{q}(J) .
\end{gathered}
$$


The eigenvalues of Eq. (267) can be written in terms of the $\Lambda_{\mathrm{m}, \mathrm{p}}^{\mathrm{q}}$ as

$$
\Omega_{q, m}+q \dot{v}_{s}=\frac{q e I_{o}}{E_{o}} z_{e f f}^{(q, m)}
$$

where the effective impedance is

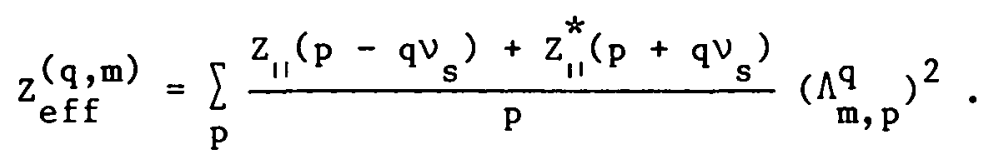

The eigenvalues and eigenfunctions of Eq. (267) depend on $g_{0}(J)$ and on $\mathrm{Z}(\omega)$. We do not have any general solution of Eq. (267). A solution has been found, however, for the case of a parabolic charge distribution and constant $Z_{11}(\omega) / \omega, Z_{11}(\omega) / \omega=L_{\omega}$. (19) Although this case per se is not very interesting, since all the eigenvalues are real and the beam is stable, it can be used to evaluate the complex eigenvalues when the impedance is of the form

$$
\frac{Z_{11}(\omega)}{\omega}=L_{\omega}+\frac{Z_{1}(\omega)}{\omega}
$$

with

$$
\left|\frac{z_{1}(\omega)}{\omega}\right| \ll L_{\omega}
$$

When Eqs. (273) and (274) are satisfied we can use perturbation methods to write the eigenfunctions and eigenvalues as

$$
\begin{gathered}
F_{m}^{q}(J)=F_{0, m}^{q}(J)+F_{1, m}^{q}(J), \\
\Omega_{q, m}=\Omega_{q, m}^{(0)}+\Omega_{q, m}^{(1)},
\end{gathered}
$$

with $\Omega_{q, m}^{(o)}$ obtained for $z_{1}=0$ and 


$$
\Omega_{q, m}^{(1)}=\frac{q e I_{o}}{E_{0}} \sum_{p} \frac{z_{1}\left(p-q \nu_{s}\right)+z_{1}^{*}\left(p+q \nu_{s}\right)}{p}\left(\Lambda_{o, m, p}^{q}\right)^{2}
$$

and the $\Lambda_{\mathrm{o}, \mathrm{m}, \mathrm{p}}^{\mathrm{q}}$ evaluated with the unperturbed eigenfunctions.

Let us now find the zeroth-order solution. We assume

$$
g_{0}(J)=\frac{3}{4 \pi} \frac{2 \eta N}{v_{s} \phi_{L}^{2}}\left\{1-\frac{2 \eta J}{v_{s} \phi_{L}^{2}}\right\}^{\frac{1}{2}}
$$

corresponding to a longitudinal density

$$
\lambda_{0}(\phi)=\frac{3 N}{4 \phi_{L}}\left\{1-\frac{\phi^{2}}{\phi_{L}^{2}}\right\} .
$$

With this choice of $g_{0}(J)$ one has $(19)$

$$
\Lambda_{o, m, p}^{q}=B_{q, m} \frac{J_{m+\frac{1}{2}}\left(p \phi_{L}\right)}{\left(p \phi_{L}\right)^{\frac{1}{2}}}, \quad m=q+2, q+4, \ldots,
$$

with

$$
\mathrm{B}_{\mathrm{q}, \mathrm{m}}^{2}=\frac{3}{8} \frac{\mathrm{Nr}}{v_{s}}(2 \mathrm{~m}+1) \frac{(\mathrm{m}+\mathrm{q}-1) ! !(\mathrm{m}-\mathrm{q}-1) ! !}{(\mathrm{m}+\mathrm{q}) ! !(\mathrm{m}-\mathrm{q}) ! !}
$$

and the eigenvalues can be written as

$$
\begin{gathered}
q \nu_{s}+\Omega_{q, m}^{(o)}=\frac{e I_{o} \eta}{E \nu_{s}} z_{q, m}^{(0)} \frac{q(m+q-1) ! !(m-q-1) ! !}{(m+q) ! !(m-q) ! !}, \\
\Omega_{q, m}^{(1)}=\frac{e{ }_{o}{ }^{n}}{E \nu_{s}} z_{q, m}^{(1)} \frac{q(m+q-1) ! !(m-q-1) ! !}{(m+q) ! !(m-q) ! !},
\end{gathered}
$$


with

$$
\begin{gathered}
z_{q, m}^{(0)}=3 \pi \frac{\omega_{0} L_{w}}{\phi_{L}^{3}}, \\
z_{q, m}^{(1)}=\frac{3}{8}(2 m+1) \int_{-\infty}^{+\infty} d y\left(\frac{J_{m+\frac{1}{2}}(y)}{y}\right)^{2} z_{1}\left(\left(y-q v_{s} \phi_{L}\right) \frac{\omega_{o}}{\phi_{L}}\right) .
\end{gathered}
$$

The formulae (284) and (285) have been widely used to evaluate the coupling impedance for a single-bunch slow instability. It must be remembered, however, that Eq. (285) can be applied only for an impedance of the form (273), (274) and that for an arbitrary impedance the result (285) is not generally valid. In particular, since the term $\mathrm{L}_{\omega}$ in $\mathrm{Eq}$. (273) produced by smooth conducting walls is very small for high energy beams, the formula (285) can be only approximately applied in frequency regions where the broad-band impedance is slowly varying.

To evaluate approximately the threshold for this instability we can compare the coherent frequency shift, whose main contribution is given by Eq. (282), with the frequency spread produced by the nonlinear part of the rf force, $\Delta \Omega_{\mathrm{L}}$,

$$
\Delta \Omega_{L}=-\frac{1}{8} B^{2} v_{S} \omega_{o} \phi_{L}^{2}
$$

Assuming $\Delta \Omega_{\mathrm{T}} \simeq \Omega_{\mathrm{q}, \mathrm{m}}^{(\mathrm{o})}$ we obtain a relationship between the current and the bunch length, defining the equilibrium conditions. It is interesting that the threshold, or equilibrium current, Ich, io a vory etrong function of the hunch 1 ength. In fact, from Eqs. (286), (282), and (284) we have

$$
\mathrm{I}_{\mathrm{th}}=\phi_{\mathrm{L}}^{5}
$$

\subsection{The high trequency instability}

When the instability rise time is shorter than the synchrotron period we cannot apply the results of the previous sections. Use of the approximate expression (249) in the Hamiltonian (247) is not justified, and the full expression (248) has to be used.

In this case all synchrotron modes $e^{i q \zeta}$ are coupled. This coupling plays an important role in the fast instability, and it is convenient, in studying this problem, to use an approach different from that used in the slow instability case. $(20)$ 
We use directly the distribution function (246) and the Hamiltonian (247). We consider again the case of a single bunch, and assume that $U_{\mathrm{NL}}=0$ and that the field produced by the equilibrium distribution causes a change only in $v_{s}$. In this case we call sewlile Llie Hatuillumian (247) as

$$
\overline{\mathrm{H}}=-\nu_{\mathrm{s}} \omega_{\mathrm{o}} \mathrm{J}+\mathrm{U} \mathrm{e}^{-\mathrm{i} \omega_{\mathrm{o}} \Omega \mathrm{t}}
$$

with

$$
U=\frac{e \omega_{0} I_{o}}{E_{0}} \sum_{p} \frac{Z_{11}(p+\Omega)}{p} \Lambda_{p} e^{i p \phi}
$$

and

$$
\Lambda_{\mathrm{p}}=\frac{1}{2 \pi} \int \mathrm{dJd} \zeta g_{1}(\mathrm{~J}, \zeta) \mathrm{e}^{-i p \phi(J, \zeta)}
$$

and with $\phi(\mathrm{J}, \zeta)$ given by Eq. (88).

The linearized Vlasov equation cán now be written as

$$
i \omega_{0} \Omega_{g_{1}}+\omega_{0} \nu_{s} \frac{\partial g_{1}}{\partial \zeta}=-\frac{\partial U}{\partial \zeta} \frac{\partial g_{0}}{\partial J} \text {. }
$$

The solution of Eq. (291) can be written, using the periodicity in $\zeta$, as

$$
g_{1}(J, \zeta)=-\frac{1}{\omega_{0} \nu_{s}} \frac{\partial g_{0}}{\partial J} \frac{e^{-i \bar{\Omega} \zeta}}{e^{2 \pi i \bar{\Omega}}-1} \int_{\zeta}^{\zeta+2 \pi} d \zeta^{\prime} \frac{\partial U\left(J, \zeta^{\prime}\right)}{d \zeta^{\prime}} e^{i \bar{\Omega} \zeta^{\prime}}
$$

where

$$
\bar{\Omega}=\Omega / \nu_{s}
$$

By substituting Eq. (292) in Eq. (290) we can reduce the integral equation (292) to a linear algebraic system

$$
\Lambda_{\mathrm{p}}=\sum_{\mathrm{q}=-\infty}^{+\infty} \mathrm{T} \mathrm{pq}_{\mathrm{q}}
$$

with 


$$
\begin{gathered}
T_{p q}=\frac{i_{0} I_{o}}{2 \pi \nu_{s} E_{0}} \frac{z_{11}(q+\Omega)}{e^{2 \pi i \bar{\Omega}}-1} \\
\times \int_{0}^{\infty} d J \frac{\partial g}{\partial J}\left(\frac{2 n J}{v_{s}}\right)^{3 \frac{1}{2}} \int_{0}^{2 \pi} d \zeta d \bar{\zeta} \sin (\zeta+\bar{\zeta}) e^{i i \bar{\Omega} \bar{\zeta}-i p \phi(J, \zeta)+i q \phi(J, \zeta+\bar{\zeta})} .
\end{gathered}
$$

The solutions of our problem are the values of $\Omega$ for which the equation

$$
\operatorname{det}\left|\delta_{\mu, \varphi}-T_{\mu \varphi}\right|=0
$$

is satisfied.

We emphasize here that, contrary to the coasting beam case, for a bunched beam all frequency components $q \omega_{0}$ are coupled. Also all synchrotron modes $g_{1}(\mathrm{~J}, \zeta) \simeq \mathscr{\xi}_{1}(\mathrm{~J}) \mathrm{e}^{i q \zeta}$ are coupled, contrary to the slow instability case. In this coupling of all modes lies the difficulty of solving the fast blowup, bunched beam case.

To study Eq. (294) we use the following approach. We consider only high frequency perturbations, i.e., the case

$$
\mathrm{q} \sigma_{\phi} \gtrsim 1
$$

where $\sigma_{\phi}$ is the r.m.s. bunch length in units of machine radius. Then we consider the fast blowup case

$$
\omega_{0} \gg|\Omega| \omega_{0} \gg v_{s} \omega_{0}
$$

Using these two conditions we simplify the expression of the matrix element (295) by assuming $z_{11}(q+\Omega) \simeq z_{11}(q)$ and by evaluating the integral over $\bar{\zeta}$ in the 1 imit $\operatorname{Im} \Omega \rightarrow+\infty$. We also rewrite the integration over $J$ and $\zeta$ as an integral nver $\varepsilon$ and $\phi$ to obtain $(20)$

$$
T_{p q} \simeq-\frac{i e I_{o}}{2 \pi E_{0}} z_{11}(q) \int d \varepsilon d \phi e^{i(q-p) \phi} \frac{\partial g_{o} / \partial \varepsilon}{\Omega+q \eta \varepsilon}
$$


We now restrict ourselves to the case of a Gaussian bunch

$$
\begin{gathered}
g_{0}=\lambda(\phi) G(\varepsilon), \\
\lambda(\phi)=\frac{1}{\sqrt{2 \pi} \sigma_{\phi}} \mathrm{e}^{-\phi^{2} / 2 \sigma_{\phi}^{2},} \\
G(\varepsilon)=\frac{1}{\sqrt{2 \pi} \sigma_{\varepsilon}} \mathrm{e}^{-\varepsilon^{2} / 2 \sigma_{\varepsilon}^{2}} .
\end{gathered}
$$

We also define

$$
\lambda_{\mathrm{n}}=\frac{1}{2 \pi} \int \mathrm{d} \phi \lambda(\phi) \mathrm{e}^{\mathrm{in} \phi}=\frac{1}{2 \pi} \mathrm{e}^{-\mathrm{n}^{2} \sigma_{\phi}^{2} / 2} .
$$

The matrix element can now be written as

$$
T_{p q}=\frac{e I_{o}}{E_{o} \eta} \frac{Z_{11}(q)}{q} \lambda_{q-p} \int d \varepsilon \frac{\partial G / \partial \varepsilon}{\Omega / q \eta+\varepsilon}
$$

or, using Eq. (302), as

$$
T_{p q}=-\frac{2 \pi e I_{o}}{n E_{o} \sigma_{\varepsilon}^{2}} \frac{z_{11}(q)}{q} \lambda_{q-p} h\left(-\frac{\Omega}{q \sigma_{\phi} \nu_{s}}\right)
$$

with

$$
h(y)=\int_{0}^{+\infty} d x x e^{-x^{2} / 2-i x y} .
$$

This function has the property that

$$
\begin{gathered}
\mathrm{h}_{1}(0)=1, \\
|\mathrm{~h}(\mathrm{y})| \leq 1 \quad \text { if } \mathrm{y} \neq 0 \text {, Imy }<0,
\end{gathered}
$$

so that it has a maximum for $y=0$ and decreases when $y$ increases. 
It is interesting that the matrix element (304) or (305) can be used also to describe the limiting case of a coasting beam by taking the other limit $\sigma_{\phi} \rightarrow \infty$ or

$$
\lambda_{\mathrm{n}-\mathrm{m}}=\delta_{\mathrm{n}, \mathrm{m}}
$$

In this case the matrix $\mathrm{T}$ is diagonal, and we obtain the usual coasting beam dispersion relation (206)

$$
1=-\frac{e I_{o}}{E \eta} \frac{Z_{11}(q)}{q} \int d \varepsilon \frac{\partial G / \partial \varepsilon}{\Omega / q \eta+\varepsilon} .
$$

For a bunched beam we have to solve the more complicated equation (294) with $\mathrm{T}_{\mathrm{m}, \mathrm{n}}$ given by $\mathrm{Eq}$. (305). We can obtain a simple but approximate solution if we make the approximation that the impedance is large only near a frequency $q_{o}$ and is nearly constant over the range $q_{o}-N_{b}, q_{o}+N_{b}$ where $N_{b} \backsim 1 / \sigma_{\phi}$ defines the range over which the bunch form factor $\lambda_{n}$ remains near one. In this simple model, we can rewrite $\mathrm{Eq}$. (305) as

$$
T_{p q}^{*} \simeq-\frac{e I_{o}}{E_{o} n \sigma_{E}^{2}} \frac{z_{11}\left(q_{o}\right)}{q_{o}} h\left(-\frac{\Omega}{q_{o} \sigma_{\phi} \nu_{s}}\right) e^{-(q-p)^{2} \sigma_{\phi}^{2} / 2} .
$$

The eigenvectors of the matrix $\mathrm{T}_{\mathrm{pq}}^{*}$ are of the form

$$
v_{p}=e^{i \alpha p}
$$

and the corresponding eigenvalues are obtained from the dispersion relation

$$
1=-\frac{e I_{o}}{E_{0} n \sigma_{\varepsilon}^{2}} \frac{z_{11}\left(q_{o}\right)}{q_{o}} h\left(-\frac{\Omega(\alpha)}{q_{o} \sigma_{\phi} \nu_{s}}\right) \sum_{n=-\infty}^{+\infty} e^{-\left(n^{2} \sigma_{\phi}^{2} / 2\right)+i n \alpha} .
$$

Since $|h(x)| \leq 1$ and the sum over $n$ in Eq. (312) has a maximum for $\alpha=0$, we can obtain a solution of Eq. (312) only if the condition 


$$
\frac{e I_{o}}{E_{o} n \sigma_{\varepsilon}^{2}}\left|\frac{z_{11}\left(q_{o}\right)}{q_{o}}\right| \sum_{n=-\infty}^{+\infty} e^{-\left(n^{2} \sigma_{\phi}^{2} / 2\right)} \geq 1
$$

is satisfied. Note that the sum over $\mathrm{n}$ in Eq. (313) is of the order of $1 / \sigma_{\phi}$, so that this condition depends on $I_{0} / \sigma_{\phi}$, i.e., the peak current.

If the condition (313) is not satisfied, we contradict our initial assumption $\left|\Omega / \nu_{s}\right|>1$. On the other hand if we satisfy (313) we can find a solution of Eq. ( 312 ) with $\left|\Omega / \nu_{s}\right|>1$, consistent with the way we have derived Eq. (305). This means that we can have a fast blowup only if the beam current is such as to satisfy Eq. (313) and that at lower beam current there is no possibility of a fast blowup.

It is also interesting that for a given $\Omega / \nu_{0} \omega_{0}$, the function $h\left(-\Omega / q_{0} \sigma_{\phi} \nu_{s}\right)$ is larger when $q_{0} \sigma_{\phi}>\left|\Omega / \nu_{s}\right|>1$, so that a high frequency impedance is more effective in producing a fast blowup.

Equation (313) can be used to define a threshold curve $I_{\rho}=f\left(\sigma_{\phi}\right)$. For a bunch, since $\sigma_{\varepsilon} \simeq \sigma_{\phi}$, this curve is of the form

$$
I_{t h} \simeq \sigma_{\phi}^{3}
$$

Comparing Eq. (314) with the similar condition (287) for slow instabilities, we see that at small current the condition (287) is dominant and single-mode (slow) instabilities occur. With increasing current, the microwave (fast) instability becomes dominant.

\section{Transverse Instabilities of a Coasting Beam}

In this section we start the discussion of transverse instabilities by considering the simplest case, i.e., that of a coasting beam. (21) For a coasting beam the equilibrium state has a uniform distribution in azimuth and no rf system is present. The equations of motion can be simplified by assuming $\nu_{s}=0$. (101) as

Using action-angle variables we obtain the Hamiltonian from Eqs. (100) and

$$
\overline{\mathrm{H}}=\nu_{0} \omega_{0}\{I(1+P C), D(I)\}-\frac{1}{2} \eta \omega_{0} \varepsilon^{2}-F(\psi, t)\left(\frac{2 I}{v_{0} \omega_{0}}\right)^{\frac{1}{2}} \cos \psi .
$$


To write the distribution function, we follow Eq. (115) and neglect for simplicit.y the inessential variables $x, \dot{x}$. In the coasting beam case $\varepsilon$ is constant; there is no modulation of the betatron frequency due to synchrotron oscillations.

We now assume explicitly

$$
f=\frac{N}{R}\left\{\frac{1}{2 \pi} g_{0}(\varepsilon) h_{0}(I)+f_{1}^{P}(I, \Psi, \varepsilon) e^{i p \phi-i v \omega_{0} t}\right\},
$$

having selected only one of the Fourier harmonics in $\phi$ to describe the perturbation. It will be seen that this choice is justified because the harmonics with different $\mathrm{p}^{\prime}$ 's do not interact and Eq. (316) is an eigenfunction in $\phi$ of our problem. In the same way we select only one of the harmonics of $F$ in Eq. (173):

$$
F=\frac{i e 1_{0} B}{2 \pi R} D_{p} Z_{1}(p+\nu) e^{i p \phi-i \nu \omega_{0} t}
$$
tion 3)

If we want to study the solution with $\nu \simeq \nu_{0}$ we can also assume (as in Sec-

$$
\mathrm{f}_{1}^{\mathrm{p}} \simeq \tilde{f}_{1}^{\tilde{p}_{1}} \mathrm{e}^{\mathrm{i} \psi}
$$

and obtain the linearized Vlasov equation

$$
\begin{gathered}
\left\{\nu-\nu_{0}-\nu_{0}\left(D^{\prime}(I)+\left(\xi-\eta+\frac{p \eta}{\nu_{0}}\right) \varepsilon\right)\right\} \tilde{f}_{1}^{v_{p}}(I, \varepsilon) \\
=\frac{i e I 0}{8 \pi^{2} E_{0}} D_{p} Z_{1}(p+\nu)\left(\frac{2 I}{\nu_{0} \omega_{0}}\right)^{\frac{1}{2}} g_{0}(\varepsilon) \frac{\partial h_{0}(I)}{\partial I},
\end{gathered}
$$

having used Eq. (92) to express P.

The dipole moment in Eq. (319) is given by Eqs. (159) and (98) as

$$
D_{p}=\int d \varepsilon d I d \psi f_{1}^{(p)}(I, \psi, \varepsilon)\left(\frac{2 I}{v_{0} \omega_{0}}\right)^{\frac{1}{2}} \cos \psi
$$


and, by using (318), as

$$
\mathrm{D}_{\mathrm{P}}=\pi \int \mathrm{d} \varepsilon \mathrm{dI}\left(\frac{2 \mathrm{I}}{v_{0} \omega_{0}}\right)^{\frac{1}{2}} \stackrel{\mathrm{f}}{\mathrm{f}}_{1}^{\mathrm{p}}(\mathrm{I}, \varepsilon) .
$$

From Eqs. (319) and (321) we obtain the dispersion relation(21)

$$
1=\delta \nu_{p} \int \mathrm{d} \varepsilon \mathrm{dI} \frac{\operatorname{Ig}_{0}(\varepsilon) \frac{\partial \mathrm{h}_{0}(I)}{\partial I}}{\nu-\nu_{0}-\nu_{0}\left(D^{\prime}(I)+\left(\xi-\eta+\frac{\mathrm{p} \eta}{\nu_{0}}\right) \varepsilon\right)}
$$

with

$$
\delta \nu_{p}=\frac{i e I_{0} R}{4 \pi \beta E_{0} \nu_{0}} z_{1}\left(p+\nu_{0}\right)
$$

giving the coherent frequency shift. In Eq. (323) we have evaluated the impedance, to zero order, for $\nu=\nu_{0}$.

In the simple case in which $D^{\prime}(I)=0$ and $g_{0}(\varepsilon)=\delta(\varepsilon)$ the solution of the dispersion integral is

$$
\nu-v_{0}=\delta v_{p}
$$

In the general case the value of $\nu$ depends on the energy and the amplitude distribution. In systems like proton storage rings, the energy dependent term, in the denominator of the dispersion integral, can be larger than the amplitude dependent term. In this case Eq. (322) can be written in the simpler form

$$
1=\delta \nu_{p} \int d \varepsilon \frac{g_{0}(\varepsilon)}{\nu_{0}-\nu+\nu_{o}\left(\xi-n+\frac{p \eta}{\nu_{0}}\right) \varepsilon} .
$$

This is the same type of dispersion relation discussed in Section 2, and we can follow the same evaluation to arrive at the stability condition

$$
\left|\delta v_{p}\right| \simeq \Delta v_{p}
$$

with 


$$
\Delta \nu_{P}=\nu_{0}\left(\xi-\eta+\frac{p \eta}{\nu_{0}}\right) \varepsilon_{M}
$$

giving the frequency spread in terms of the energy spread $\varepsilon_{M}$.

It is interesting that, for a given energy spread, the frequency spread is determined, according to Eq. (327), by the machine chrumaticity, $\xi$, the frequency slip factor, $\eta$, and the mode number, $p$. In particular one can define a mode number, $p^{*}$, such that $\Delta v$ is a minimum. Then, if we satisfy Eq. (326) for $p=p^{*}$, the beam is stable for all other values of $p$, that is, for all coherent dipole modes.

\section{Transverse Instabilities of Bunched Beams}

11.1. The Vlasov equation for the transverse oscillations of a bunched beam

To obtain the Vlasov equation we use action-angle coordinates. The Hamiltonian for bunch $l$ is obtained from Eq. (109),

$$
\begin{aligned}
\bar{H}^{(\ell)} & =\nu_{0} \omega_{0}\left\{\left(1+P \varepsilon_{\ell}\right) I_{\ell}+n\left(I_{\ell}\right)\right\} \\
& -\nu_{s} \omega_{0} J_{\ell}-F\left(J_{\ell}, \zeta_{\ell}, t\right)\left(\frac{2 I_{\ell}}{\nu_{0} \omega_{0}}\right)^{\frac{1}{2}} \cos \psi_{\ell}
\end{aligned}
$$

where, for simplicity, we have assumed the energy to be above transition, $n>0$. The collective force is given by Eqs. (175) and (177).

The distribution function for bunch $\dot{x}$, neglecting the inessential vartables $x, \dot{x}$, can be written as

$$
f^{(l)}=\frac{N}{R}\left\{f_{0}^{(l)}(I, J)+f_{1}^{(l)}(I, \psi, J, \zeta) \ell^{-i \omega_{0} L}\right\} .
$$

. The linearized Vlasov equation is

$$
\begin{aligned}
-i \omega_{0} f_{1}^{(l)} & +\nu_{0} \omega_{0}\left\{1+P \varepsilon+D^{\prime}(I)\right\} \frac{\partial f_{1}^{(l)}}{\partial \psi}-\nu_{0} \omega_{0} \frac{\partial f_{1}^{(l)}}{\partial \zeta} \\
& =\frac{c^{2} F^{(l)}}{E_{0}} l^{i \omega_{0} t}\left(\frac{2 I}{\nu_{0} \omega_{0}}\right)^{\frac{1}{2}} \sin \psi \frac{\partial f_{0}^{(l)}}{\partial I}
\end{aligned}
$$


with

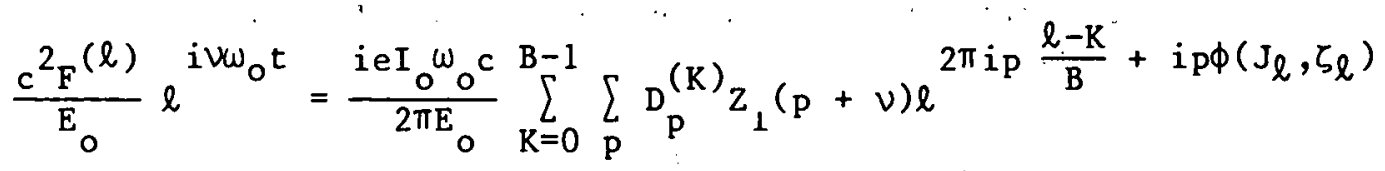

and

$$
\begin{gathered}
\mathrm{D}_{\mathrm{p}}^{(\mathrm{K})}=\frac{1}{2 \pi} \int\left(\frac{2 I}{v_{0} \omega_{0}}\right)^{\frac{1}{2}} \cos \psi \mathrm{f}_{1}^{(\mathrm{K})}(\mathrm{I}, \psi, \mathrm{J}, \zeta) \ell^{-\mathrm{i} \phi \phi(\mathrm{J}, \zeta)} \mathrm{dId} \psi \mathrm{dJ} \mathrm{d} \zeta, \\
\phi(\mathrm{J}, \zeta)=\left(\frac{2 \eta \mathrm{J}}{v_{s}}\right)^{\frac{1}{2}} \cos \zeta \ldots
\end{gathered}
$$

where we have used Eqs. (175), (177), and (88).

As discussed in Sections 2 and 3 we now look for a solution $\nu \simeq \nu_{0}$ and assume that $\mathrm{f}_{1}(\mathrm{I}, \Psi, \mathrm{J}, \zeta) \simeq \mathrm{f}_{1}(\mathrm{I}, \mathrm{J}, \zeta) \mathrm{e}^{i \psi}$. Then $\mathrm{Eq} \cdot(330)$ becomes

$$
\begin{aligned}
& \left\{\nu-\nu_{0}\left(1+P_{\varepsilon}+D^{\prime}(I)\right)\right\} \tilde{f}_{1}^{\tilde{s}(l)}-i \nu_{s} \frac{\partial \hat{f}(l)}{\partial \zeta} \\
& =\frac{i e I_{0} c}{4 \pi E_{0}}\left(\frac{2 I}{v_{0}^{\omega}{ }_{0}}\right)^{\frac{1}{2}} \frac{\partial f_{o}(I, J)}{\partial I} \sum_{K=0}^{B-1} \sum_{p} D_{p}(K) Z_{\perp}(p+\nu) e^{2 \pi i p} \frac{l-K}{B}+i p \phi(J, \zeta)
\end{aligned}
$$

where

$$
D_{P}^{(K)}=\frac{1}{2} \int\left(\frac{2 I}{v_{0} \omega_{0}}\right)^{\frac{1}{2}}{\underset{f}{f}}_{1}^{(K)}(I, J, \zeta) e^{-i p \phi(J, \zeta)} d I d J d \zeta .
$$

As in the case of longitudinal oscillations we do not have a general solution of Eq. (334). We can again divide the parameter space of this problem into a few regions where we can obtain approximate solutions. Since the betatron period is shorter than the revolution period, we can always assume that the coherent tune shift is small compared with $\nu_{0}$ and use the same classification into slow or fast instability introduced in Eqs. (242) and (243), comparing the coherent tune shift with the synchrotron tune. In the same way we can also use the long or. short wavelength approximation, shown in Eqs. (244) and (245). 


\subsection{The slow instabilities for the transverse case}

We will discuss the slow instabilities, neglecting Landau damping, $D^{\prime}(I)=0$, to simplify our problem. It is convenient to introduce a local dipole moment, defined along a bunch,

$$
v^{(K)}(J, \zeta)=\frac{1}{2} \int_{0}^{\infty} d I\left(\frac{2 I}{v_{0} \omega_{0}}\right)^{\frac{1}{2}} \tilde{f}_{1}(K)(I, J, \zeta)
$$

so that, from Eq. (335),

$$
D_{p}^{(K)}=\int d J d \zeta v^{(K)}(J, \zeta) e^{-i p \phi(J, \zeta)}
$$

From Eq. (334), multiplying by $\left(2 I / \nu_{0} \omega_{0}\right)^{\frac{3}{2}}$ and integrating over $I$, we have

$$
\begin{aligned}
& \left(v-u_{0}\left(1+p_{\varepsilon}\right)\right) v^{(l)}\left(. I, r_{0}\right)-i v_{s} \frac{\partial v^{(l)}(J, \zeta)}{\partial \zeta} \\
& =-\frac{i e I_{0}^{R}}{4 \pi B \nu_{0}^{E} E_{0}} g_{0}(J) \sum_{K=0}^{B-1} \sum_{p} D_{P}^{(K)} Z_{L}(p+\nu) e^{2 \pi i p \frac{\ell-K}{B}+i p \phi(J, \zeta)}
\end{aligned}
$$

where

$$
\varepsilon_{0}(. T)=\int_{0}^{\infty} f_{0}(T, . J) d T
$$

gives the unperturbed longitudinal distribution. We can simplify Eq. (338) by introducing the coupled bunch normal modes

$$
\begin{aligned}
& v^{(\ell)}(J, \zeta)=v_{s}(J, \zeta) e^{2 \pi i s \ell / B}, \quad s=0,1, \ldots, B-1, \\
& D_{s, p}=\int \operatorname{dJd} \zeta v_{s}(J, \zeta) e^{-i p \phi}, \quad s=0,1, \ldots, B-1,
\end{aligned}
$$

obtaining 


$$
\begin{gathered}
\left(\nu-\nu_{0}\left(1+P_{\varepsilon}\right)\right) v_{s}(J, \zeta)-i v_{s} \frac{\partial v_{s}(J, \zeta)}{\partial \zeta} \\
=-\frac{i e I_{0} B R}{4 \pi B v_{0} E_{0}} g_{0}(J) \sum_{n} D_{s, n B+s} Z_{1}(n B+s+\nu) e^{i(n B+s) \phi} .
\end{gathered}
$$

As in the case of coupled bunch longitudinal instabilities, the frequency spectrum is a multiple of the bunch revolution frequency, plus the mode number $s$, plus the coherent betatron frequency $v$.

The solution of Eq. (342), periodic in $\zeta$, is

$$
\begin{aligned}
& v_{s}(J, \zeta)=-\frac{i e I_{o} B R}{4 \pi B E_{0} \nu_{o} \nu_{s}} g_{o}(J) \sum_{n} D_{s, n B+s} Z_{1}(n B+s+\nu) \times \frac{1}{e^{2 \pi i\left(\nu-\nu_{o}\right) / v_{s}}-1} \\
& \times \int_{\zeta}^{\zeta+2 \pi} d \zeta^{\prime} e^{i(n B+s) \phi\left(J, \zeta^{\prime}\right)+i \frac{\nu-\nu}{\nu_{s}}\left(\zeta^{\prime}-\zeta\right)-i \bar{\nu}\left(\phi\left(J, \zeta^{\prime}\right)-\phi(J, \zeta)\right)}
\end{aligned}
$$

with

$$
\bar{v}=\nu_{0} P / \eta=\nu_{0}\left(\frac{\xi}{\eta}-1\right)
$$

We now expand $e^{i \mu \phi}$ in its Fourier harmonics to obtain

$$
\begin{gathered}
v_{s}(J, \zeta)=-\frac{i e I_{0} R}{4 \pi B E_{o} \nu_{o}} g_{o}(J) \sum_{n} D_{s, n B+s} Z_{1}(n B+s-v) \\
\times \sum_{p, q=-\infty}^{+\infty} \frac{i^{p+q}}{v-v_{o}+q v_{s}} J_{p}\left\{\bar{v}\left(\frac{2 n J}{v_{s}}\right)^{\frac{1}{2}}\right\}_{J_{q}}\left\{(n B+s-\bar{v})\left(\frac{2 \eta J}{\nu}\right)^{\frac{1}{2}}\right\} e^{i(p+q) \zeta} .
\end{gathered}
$$


On this equation we can perform the slow instability approximation by taking the average over $\zeta$, i.e., neglecting all fast oscillating terms and keeping only the term $p+q=0$.

The approximate equation for the average value, $\bar{v}_{s}(J)$, of $v_{s}(J, \zeta)$ is

$$
\begin{aligned}
& \bar{v}_{s}(J)=-\frac{i e I_{0} R}{4 \pi B E_{o} \nu_{o}} g_{o}(J) \sum_{n} D_{s, n B+s} Z_{1}(n B+s+\nu) \\
& \times \sum_{q} \frac{(-1)^{q}}{v-\nu_{0}+q \nu_{s}} J_{q}\left\{\bar{v}\left(\frac{2 n J}{\nu_{s}}\right)^{\frac{1}{2}}\right\}_{J}\left\{(n B+s-\bar{v})\left(\frac{2 n J}{v_{s}}\right)^{\frac{1}{2}}\right\}
\end{aligned}
$$

with

$$
D_{s, p}=2 \pi \int_{\Omega}^{\infty} d J \bar{v}_{s}(J) J_{o}\left\{p\left(\frac{2 \eta J}{v_{s}}\right)^{\frac{1}{2}}\right\}
$$

Since we have made the assumption that the coherent tune shift is small compared with the synchrotron frequency, we can obtain a solution of Eq. (346) to first order in $\left(v-v_{0}\right) / \nu_{s}$ by keeping only the $q=0$ term on its $r . h . s$. The equation for the slow instability is then

$$
\begin{gathered}
\bar{v}_{s}(J)=-\frac{i e I_{0} R}{4 \pi \nu_{0} \beta E_{0}} g_{0}(J) J_{0}\left\{\bar{v}\left(\frac{2 n J}{v_{s}}\right)^{\frac{1}{2}}\right\} \times \frac{1}{v-v_{0}} \\
\times \sum_{n} D_{s, n B+s} J_{0}\left\{(n B+s-\bar{v})\left(\frac{2 n J}{v_{s}}\right)^{\frac{1}{2}}\right\} z_{\perp}(n B+s+v) .
\end{gathered}
$$

This equation is still very complicated, and it is convenient to obtain some simplified and approximate solution to provide some understanding of the transverse instabilities.

\subsection{Rigid bunches and the coupled bunch instabilities}

For a bunch of length $\phi_{\mathrm{L}}$ the quantity $\bar{v} \phi_{\mathrm{L}}$ measures the change in betatron frequency from the head to the tail of the bunch. In this section we study the case in which this change is negligible and all particles in the bunch oscillate with the same frequency, as in a point-like $\left(\phi_{L}=0\right)$ system or rigid system. Assuming $\bar{v}=0$ in Eq. (348) we obtain the equation 


$$
\bar{v}_{s}(J)=-\frac{i e I o_{0}^{R}}{4 \pi B E_{0} \nu_{0}} \frac{g_{0}(J)}{\nu-\nu_{0}} \sum_{n} D_{s, n B+s} Z_{1}(n B+s+\nu) J_{0}\left\{(n B+s)\left(\frac{2 \eta J}{\nu_{s}}\right)^{\frac{1}{2}}\right\}
$$

describing coupled bunch instabilities.

Equation (348) becomes very simple in the long wavelength approximation, taking $\mathrm{J}_{\mathrm{o}}\left\{\mathrm{p}\left(2 \mathrm{~nJ} / \nu_{\mathrm{s}}\right)^{\frac{1}{2}}\right\} \simeq 1$ in the expression (347) of the dipole moment $\mathrm{D}_{\mathrm{s}}, \mathrm{p}$,
which in this case does not depend on p. We obtain from Eg. (349) the which in this case does not depend on $p$. We obtain from Eg;
eigenvalue for the $s-t h$ mode of coherent oscillation, as $(22)$

$$
\nu-\nu_{0}=-\frac{i e I_{0} R}{4 \pi \beta E E_{0} \nu_{0}} z_{\text {eff }}^{s}
$$

with

$$
Z_{\text {eff }}^{s}=\sum_{n} Z_{\perp}\left(n B+s+\nu_{0}\right) F(n B+s)
$$

and

$$
F(p)=2 \pi \int_{0}^{\infty} d J g_{0}(J) J_{0}\left\{p\left(\frac{2 \eta J}{\nu_{s}}\right)^{\frac{1}{2}}\right\}
$$

The form factor $F(p)$ can be evaluated for any bunch shape. For a Gaussian bunch, $g_{0}(J)$ is given by Eq. (259) and

$$
F(p)=e^{-p^{2} \sigma^{2} \phi / 2}
$$

The eigenvalues ( 349 ) describe the $B$ collective mode of oscillations of $B$ rigid bunches. For mode $s$ the bunches oscillate with a phase difference $2 \pi s / B$.

\subsection{The head-tail instability}

We next discuss the case in which $\bar{v} \phi_{L}$ is not negligible and study the effect of the phase difference introduced within a bunch by this term. Since this effect is mainly internal to the bunch, we can simplify the problem by considering the case of a single bunch, $B=1, s=0$. We also consider only a simple impedance which does not produce an instability for a rigid bunch, for instance the case $Z_{\perp}(\omega)=$ constant, which, with use of Eqs. (351), (353), and (13), gives $\operatorname{Re} Z_{\text {eff }}=0$ and $\operatorname{Im\nu }=0$. 
Our_problem is now to study the transverse instability of a single bunch assuming $\bar{v} \phi_{\mathrm{L}}$ to be small but non zero.

Let us write Eq. (348) for $B=1, s=0$ and up to first order in $\bar{v}_{\mathrm{L}}$. Since we are considering the slow instability case, we can also assume $\left|\nu-v_{0}\right|$ $<v_{s}$, and obtain

$$
\begin{aligned}
& \bar{v}(J)=-\frac{i e I_{0}^{R}}{4 \pi B E_{0} \nu_{0}} \frac{g_{0}(J)}{v-\nu_{0}}\left\{D_{0} z_{L}(\nu)+\sum_{n>0} D_{n} J_{0}\left\{n\left(\frac{2 n J}{\nu s}\right)^{\frac{1}{2}}\right\}\left(z_{L}(n+\nu)-z_{1}^{*}(n-\nu)\right)\right. \\
& \left.+\bar{v}\left(\frac{2 \eta J}{v_{s}}\right)^{\frac{1}{2}} \sum_{n>0} D_{n} J_{1}\left\{n\left(\frac{2 \eta J}{v_{s}}\right)^{\frac{1}{2}}\right\}\left(z_{1}(n+v)+z_{1}^{*}(n-v)\right)\right\} .
\end{aligned}
$$

If we now assume $Z_{\perp}(\nu)=0$ and $z_{\perp}(\omega)=$ constant $\equiv Z_{\perp}$ we obtain the result that, if $\bar{v}=0$, then $\nu=\nu_{0}$ and the system is stable. If $\bar{v} \neq 0$, then

$$
v(J)=\frac{\delta v}{v-v_{0}} \bar{v}_{0}(J)\left(\frac{2 \eta J}{v}\right)^{\frac{1}{2}} \sum_{n>0} D_{n} J_{1}\left\{n\left(\frac{2 \eta J}{v}\right)^{\frac{1}{2}}\right\}
$$

with

$$
\delta v=-\frac{i e I_{0} R Z_{1}}{4 \pi \beta E_{v} v_{0}}
$$

If we make the additional hypothesis that we are dealing with a short bunch, so that $D_{n} \simeq n$, we can solve Eq. (355) with the help of Eq. (347), obtaining

$$
v-v_{u}=\bar{v} \delta v 2 \pi \sum_{n>0}^{\infty} \int_{0}^{\infty} d J g_{0}(J)\left(\frac{2 \eta J}{v_{s}}\right)^{\frac{1}{2}} J_{1}\left\{n\left(\frac{2 \eta J}{v_{s}}\right)^{\frac{1}{2}}\right\} .
$$

For a Gaussian bunch, with $g_{0}(J)$ given by $E q$. (259), we also have

$$
\nu-\nu_{0}=\bar{v} \delta \nu \sum_{n>0}\left(n \sigma_{\phi}^{2}\right) e^{-\left(n^{2} \sigma_{\phi} 2\right) / 2} .
$$


This result shows that the introduction of a phase shift between the head and the tail of the bunch can produce another type of instability, $(23)$ the head-tail effect.

The analysis just done of the transverse instability, as described by Eqs. (348) and (347), is much simplified. To obtain complete information we should solve the integral equation (348), finding its eigenfunction $\mathrm{V}_{\mathrm{S}}^{\mathrm{r}}(\mathrm{J})$ and eigenvalues $\lambda_{s, r}$.

The eigenfunctions provide a complete orthonormal set in the J-space with weight $W(J)$. In terms of the $\bar{V}_{S}^{r}(J)$, by rewriting (348) as

$$
\bar{v}_{s}(J)=\int_{0}^{\infty} d J^{\prime} G\left(J, J^{\prime}\right) \bar{v}_{s}\left(J^{\prime}\right)
$$

we can calculate $\nu$ from

$$
v-\nu_{0}=\lambda_{s, r} \int_{0}^{\infty} d J d J^{\prime} w(J) \bar{v}_{s}^{r}\left(J^{\prime}\right) G\left(J, J^{\prime}\right) \bar{v}_{s}^{r}(J)
$$

Thus, for Eq. (348), describing the slow transverse instability, we have an infinite set of eigenvalues, characterized by two numbers: $s$, the coupled bunch number, and $r$, the radial number in the synchrotron $(J, \zeta)$ phase space. The solution (358) gives the lowest eigenvalue for a mode in which all particles in the bunch oscillate together, similar to the rigid bunch dipole mode. According to the result (358), the stability or instability of this mode can be controlled by changing the sign of $\bar{v}$, i.e., the sign of the chromaticity $\xi$. A discussion of the higher modes $c$ an be found in the literature. $(23,24)$

The fast transverse instability has also been studied recently. (25) As in the case of longitudinal instability, in the limit of a fast blowup and a high frequency perturbation, the stability condition is given by the coasting beam condition (322) with the peak bunch current substituting for the average current in $\mathrm{Eq}$. (323). 


\section{References}

1. A.M. Sessler, IEEE Trans. Nuc1. Sci. NS-18, 1039 (1971).

2. F. Amman, IEEE Trans. Nuc1. Sci. NS-16, 1073 (1969).

3. A.M. Sessler and V. Vaccaro, Longitudinal Instabilities of Azimuthally Uniform Beams in a Circular Chamber with Wall of Arbitrary. Electrical Properties, CERN Report ISR-RF 67-2, 1967.

4. See, e.g., H.G. Hereward, The Elementary Theory of Landau Damping, CERN $65-20,1965$.

5. A discussion of the Vlasov equation can be found in many textbooks; see, e.g., T.Y. Wu, Kinetic Equations of Gases and Plasmas, Addison Wesley, Reading, Mass., 1966.

6. C. Pellegrini and A.M. Sessler, in Proc. Int. Conf. on High Energy Accelerators, p. 135, McGraw-Hi11, Cambridge, Mass., 1967.

7. See, e.g., P.M. Morse and H. Feshbach, Methods of Theoretical Physics, P. 811, McGraw-Hi11, New York, 1953.

8. See, e.g., A. Hofmann, K. Hübner, and B. Zotter, IEEE Trans. Nucl. Sci. NS-26, 3514 (1979); P.B. Wilson et al., IEEE Trans. Nuc1. Sci. NS-24, 1211 (1977).

9. See, e.g., V.K. Neil and A.M. Sessler, Rev. Sci. Instr. 36, 429 (1965); L.J. Laslett, V.K. Neil, and A.M. Sessler, Rev. Sci. Instr. 36, 436 (1965).

10. W. Schnel1, Transverse and Longitudinal Impedance in the ISR, CERN Report ISR-RF/70-7, 1970.

11. C.E. Nielsen and A.M. Sessler, in Proc. Int. Conf. on High Energy Accelerators, CERN, 1959 , p. 239.

12. E. Keil and W. Schnell, Concerning Longitudinal Stability in the. ISR, CERN Report ISR-TH-RF/69-48, 1969.

13. See review paper by J.L. Laclare, in Proc. Int. Conf. on High Energy Acceleracors, Cencva, Switzerland, 1980, p. 526.

14. J.M. Wang, Longitudinal Symmetric Coupled Bunch Modes, Brookhaven National Laboratory Report BNL 51302, 1980.

15. R. Littauer, C. Pellegrini, M. Sands, and B. Touschek, Longitudinal Multibunch Instabilities, Laboratori Nazionali di Frascati, Adone Internal Report, 1968; M.Q. Barton and E.G. Raka, IEEE Trans. Nucl. Sci. NS-18, 1032 (1971); see also ref. 2 . 
16. A. Renieri and F. Tazzioli, in Proc. Int. Conf. on High Energy Accelerators, Stanford, California, 1974, p. 370, C. Pellegrini and M. Sands, Coupled Bunch Longitudinal Instabilities, SLAC, PEP-258, 1977.

17. K. Robinson, CEA-11, 1956; CEAL-1010, 1964 .

18. F.J. Sacherer, IEEE Trans. Nuc1. Sci. NS-24, 1393 (1977).

19. F.J. Sacherer, Ph.D. Thesis, UCRL-18454, 1968; R. Gluckstern, in Proc. Proton Linear Accelerator Conf., Fermilab, Batavia, 1970, G. Besnier, Nuc1. Instrum. Methods 164, 235 (1979).

20. J.M. Wang and C. Pellegrini, in Proc. Int. Conf. on High Energy Accelerators, Geneva, Switzerland, 1980, p. 554.

21. L.J. Laslett, V.K. Neil, and A.M. Sessler, Rev. Sci. Instr. 36, 436 (1965).

22. E.D. Courant and A.M. Sessler, Rev. Sci. Instr. 37, 1579 (1966); E. Ferlenghi, C. Pellegrini, and B. Touschek, Nuovo Cimento 44, 253 (1966).

23. C. Pellegrini, Nuovo Cimento 64A, 477 (1969).

24. F. Sacherer; in Proc. Int. Conf. on High Energy Accelerators, Stanford, California, 1974, p. 347.

25. R.D. Ruth and J.M. Wang, IEEE Trans. Nucl. Sci. NS-26, 2405 (1981). 

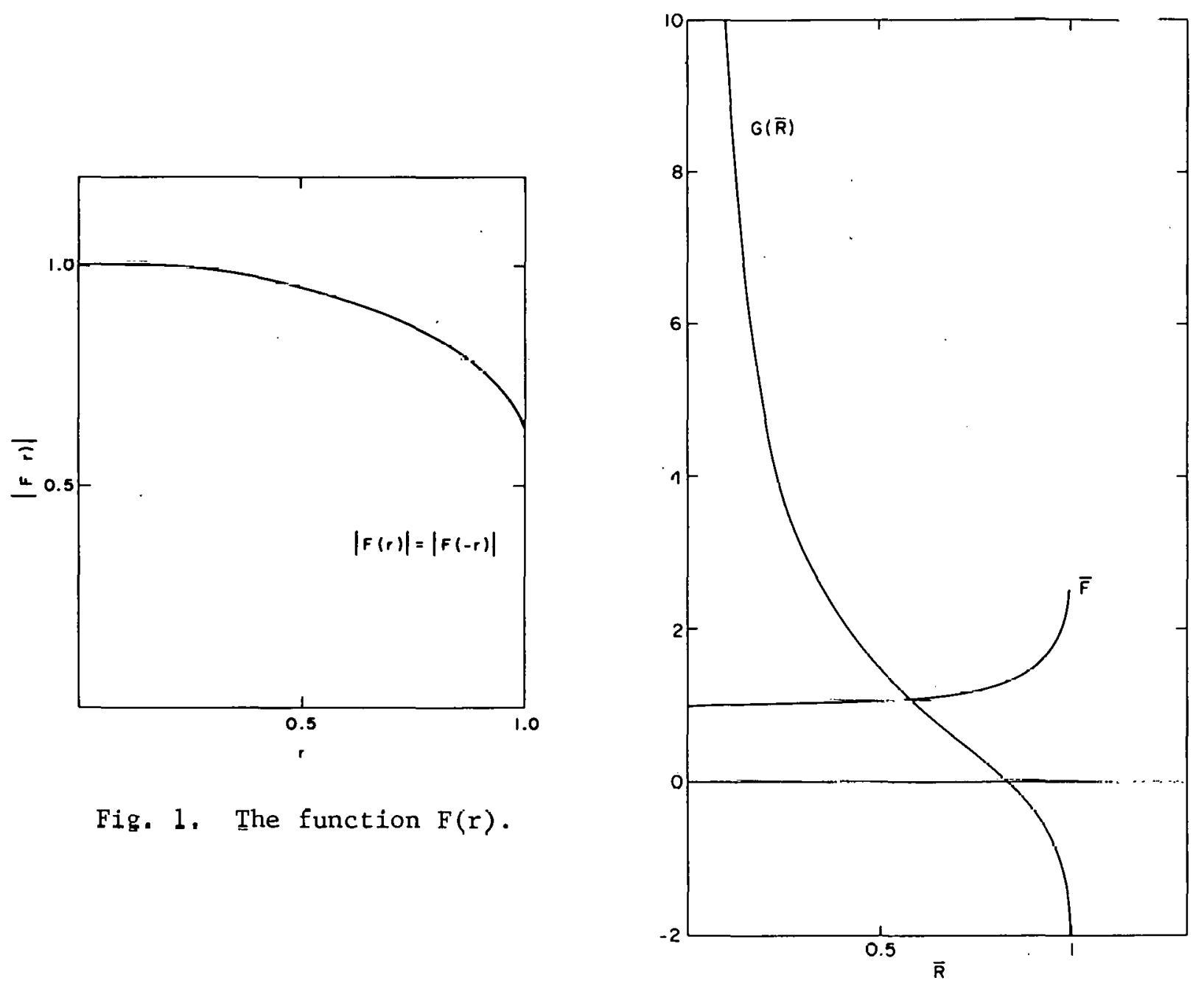

Fig. 1, The function $F(r)$.

Fig. 2. The functions $G(\bar{R})$ and $F(\bar{R})$. 